\title{
Spectral/quadrature duality: Picard-Vessiot theory and finite-gap potentials
}

\author{
Yurii V. Brezhnev
}

\begin{abstract}
In the framework of differential Galois theory we treat the classical spectral problem $\Psi^{\prime \prime}-u(x) \Psi=\lambda \Psi$ and its finite-gap potentials as exactly solvable in quadratures by Picard-Vessiot without involving special functions; the ideology goes back to the 1919 works by J. Drach. We show that duality between spectral and quadrature approaches is realized through the Weierstrass permutation theorem for a logarithmic Abelian integral. From this standpoint we inspect known facts and obtain new ones: an important formula for the $\Psi$-function and $\Theta$-function extensions of Picard-Vessiot fields. In particular, extensions by Jacobi's $\theta$-functions lead to the (quadrature) algebraically integrable equations for the $\theta$-functions themselves.
\end{abstract}

\section{Contents}

1. Introduction 2

2. Background 5

3. Integrability of equation (1) by Picard-Vessiot 8

4. Spectral/quadrature duality. An integration procedure 12

5. The $\Theta$-functons 16

6. Integration as a linearly exponential $\Theta$-extension 19

7. Integrability and differential closedness 20

8. Definition of $\theta$ through Liouvillian extension 24

9. Non-finite-gap integrable counterexamples 27

10. Concluding remarks 29

References 30

Key words and phrases. Schrödinger equation, finite-gap potentials, Picard-Vessiot theory, quadratures, Liouvillian extensions, Abelian integrals, theta-functions.

Research supported by the Federal Targeted Program under contract 02.740.11.0238 and partially by Royal Society/NATO and RFBR grant (00-01-00782).

(C)0000 (copyright holder) 


\section{Introduction}

Initially the method of finite-gap integration was developed in works [48, 29, $30,39,44,22,23]$ as a periodic generalization of the celebrated inverse scattering transform method (the soliton theory [46]). In the very first papers on this topic $[48,30,41,44,22]$ it has become clear that analogs and generalizations of the soliton potentials are to be smooth and real functions $u(x)$ in the spectral problem defined by the Schrödinger equation

$$
\Psi_{x x}-u(x) \Psi=\lambda \Psi,
$$

if the continuous spectrum of the problem consists of finitely many 'forbidden gaps' (lacunae, bands, zones, intervals [44, 39]). This explains the widely used terminology 'finite-gap', abbreviated further as FG. Ensuing development of the theory went far beyond equation (1) and took the algebro-geometric characterization $[25,37,38,24]$. Riemann surfaces and their theta-functions have become the main subject of study $[41,24,25]$. Appearance of these nontrivial objects is dictated by the very nontrivial dependence of solution to Eq. (1) upon parameter $\lambda$ and the search for this dependence is a starting and prime subject of the spectral $[44,29,30]$ and algebro-geometric ( $\Theta$-function) [37, 24] approaches. These theories are referred frequently to as the $\Theta$-function integration. Presently, one can say that the intense study of equation (1) over the last decades led to the fact that its FG-theory has been developed almost exhaustively. In this connection, it is, perhaps, not without interest to consider one more view on integration of the problem (1).

1.1. Motivation. In the early 1980 s some authors revealed the two old papers by J. Drach [20, 21] wherein equation (1) was integrated 'directly' and main results of the theory were presented in extremely condensed form. Although these works had subsequently received some mention in the literature $[26,17,46]$ with a special emphasis to the FG-theory ([8, pp. 84-85]; written by Matveev), some surprising facet is the fact that more detailed exposition of Drach's ideology has not been presented in the modern literature hitherto. The need for such exposition is apparent when taken into account that the works ${ }^{2}[20,21]$ themselves contain no any explanations or proofs. In this connection, it is pertinent to make up for this gap and sketch an appropriate theory.

The original approach by Drach is to integrate (1) as an ODE. Indeed, equation (1) is primarily a differential equation in variable $x$ even though we consider it in the algebro-geometric [37,7] or spectral context [30,44] in which the $\Psi$ is viewed, primarily, as function of $\lambda$. Anyway, in so far as the $\Psi(x ; \lambda)$ is a function of two variables, complete theory must explain this duality and therefore provide conversion between ' $x$-' and ' $\lambda$-formulae'. On the other hand, integration of linear ODEs is the subject matter of the old and well developed differential extension of the algebraic Galois theory which is variously known as the Picard-Vessiot theory and sometimes as the Lie-Kolchin theory. The main references in this topic are monographs [50, 31, 9] and classical works [51, 32]. Strange though it may seem, the explicit discussion of a direct linkage between this theory and the modern

\footnotetext{
${ }^{1}$ If parameter $\lambda$ belongs to such a lacunae (it is a line segment on the real axe $\lambda$ ), then the $\Psi$-function growths unboundedly as a function of $x$.

${ }^{2}$ After these works Drach had not longer returned to integration of linear ODEs.
} 
aspects of integrable models associated to Eq. (1) appeared comparatively recently $[45,2]$.

Correlation between the Picard-Vessiot theory and $\Theta$-function methods brings up the following question: what is the relation between these two techniques upon applying them to the linear spectral problems, say, (1)? The formal answer (commonly accepted (?)) might be the following: the $\Theta$-series (see next section for definition) is a special function solving (1). Indeed, an integration theory by PicardVessiot begins with the precise definition of a used function class [50, 32]. By this is meant that, in the strict sense, without definition of the integrability domain, any scheme based on use of the formally postulated $\Theta$-series should indeed be considered as integration in terms of special functions. We shall show that this is not the case. For example, the $\Theta$-function integration of Hamiltonian finite-dimensional nonlinear dynamical systems $\dot{q}=V(q)$ is know to be a manifestation of their algebraically invariant Liouvillian integrability. On the other hand, integrability of such systems is very well known to be related [19] to their representability through certain linear equations (Lax pairs):

$$
\{\boldsymbol{L}(q) \Psi=\lambda \Psi, \quad \dot{\Psi}=\boldsymbol{A}(q) \Psi\} \quad \Rightarrow \quad \dot{\boldsymbol{L}}=[\boldsymbol{A}, \boldsymbol{L}] \quad \Leftrightarrow \quad \dot{q}=V(q) .
$$

The 'nonlinear Hamiltonian' integrability is certain to entail the 'linear PicardVessiot' one, if only because the logarithmic derivative $\dot{\Psi} / \Psi$ is a rational function of dynamical variables. It should be noted here that the development of this ideology can serve as a basis for independent concept of integration of spectral problems at all. However, we do not touch here on such Hamiltonian systems and algebrogeometric (FG) integration of partial differential equations (PDEs). We focus only on a linear problem as such, so our main intention with this work is to show that the scheme of FG-integration of the linear spectral equation (1) should be separated into the two parts:

(1) The invariant property of equation (1) to be integrable, i. e., Lie-Kolchin's solvability of corresponding differential Galois group [32, 50].

(2) Representation of differential fields and solutions in terms of those functions which of inevitably appear in the theory. These are the $\Theta$-series. In some particular cases the series themselves satisfy the algebraically integrable Hamiltonian ODEs.

By the invariance, here and in the subsequent discussion, we shall informally mean an independence of representations by theta-functions. Notwithstanding the fact that representation of solutions requires introducing the highly nontrivial transcendental $\Theta$-objects, the integrability mechanism itself is very simple. It coincides in effect with an elementary solvability in closed form ${ }^{3}$ and thereby trivializes understanding of the major portion of the FG-theory. In other words, our main purpose is to bring the Picard-Vessiot aspects-fields, their extensions, differential Galois group, quadrature solvability, etc-into the foreground and, subsequently, to get representations for them in the FG-terms-spectral curves, variables $\gamma_{k}$, $\Theta$ 's, etc. The latter objects, to the best of our knowledge, have not received mention

\footnotetext{
${ }^{3} \mathrm{~J}$. Kovacic, in his famous work [35] on p. 4, notes: 'by a "closed-form" solution we mean, roughly, one that can be written down by a first-year calculus student'. As we shall see, this 'definition' is completely compatible with the transcendental $\Theta$-function characterization of the FG-integration of Eq. (1). Rephrasing, there is a closed form solution (Theorem 4.2) that can be verified by a direct substitution into (1) followed by use of the first-year student calculus: algebra and differentiation.
} 
in the contemporary works on the Picard-Vessiot integration of linear ODEs. See, for example, work [49, first sentence in $\S 3(\mathrm{~b})]$, monographs [50, 45, 9], and quite voluminous references therein. Partially, some fragments of the theory, in a context of the elliptic Lamé potentials $u=A \wp(x)$, can be found in book [45] and work [10]. We note also that FG-potentials are the Abelian functions [41] and Abelian extensions of differential fields were already briefly considered by Kolchin himself [33]. There is no escape from the mentioning nice applications of the Picard-Vessiot theory of Eq. (1) to the supersymmetric quantum mechanics. They appeared comparatively recently and this theme is the subject matter of recent works [1, 2].

1.2. Outline of the work. Section 2 contains the background material: a sketch of the classical Burchnall-Chaundy theory of commuting operators and its modern formulation in the language of theta-functions.

In Sect. 3 we briefly recall the needed facts from the Picard-Vessiot theory, introduce the base differential fields (Novikov's fields), and motivate their hyperelliptic extensions. Then we describe a structure of the differential Galois group for FG-potentials.

Section 4 is devoted to the quadrature (Drach) characterization of the FGintegrability and an explanation as to how the known Weierstrass theorem on an Abelian logarithmic integral performs the transition and difference between quadrature and spectral mode of getting formulae for the $\Psi$-function.

In Sect. 5 we first recall that the theta-function formulae can be derived from quadratures ones [13] and then show the necessity to represent the previous base objects in terms of theta-functions and, in particular, to introduce an important object-the 1-dimensional section of the theta-function argument with a free parameter. Owing to some differential properties of theta-functions the theory acquires very effective form in those cases when jacobians are reducible to a product of elliptic curves.

In Sect. 6 we completely pass to the theta-function representations and give an appropriate formulation to the FG-Picard-Vessiot integrability of Eq. (1). This provides a nice analogy with solubilities in the simplest integrability domains like field $\mathbb{C}$.

Section 7 explains how the theta-function reformulation of Picard-Vessiot integrability transforms into the differential closedness of the theta-functions themselves. This also gives a new treatment to the spectral parameter and a relationship of this treatment to the closedness and linearity of some of defining equations. We expound results at greater length for the cases when multi-dimensional $\Theta$ reduces to the 1-dimensional Jacobian $\theta$ 's. By way of illustration we exhibit a simplest $g=2$ non-elliptic potential.

Differential properties of $\theta$-functions described in previous section allows us to take these as a starting point for definition of the functions themselves. It turns out that such a view leads again to a Liouvillian extension but the latter is accompanied by introducing a meromorphic elliptic integral and brings up some questions about differential structures of the multi-dimensional $\Theta$. All this is expounded in Sect. 8.

In Sect. 9 we exhibit some counterexamples fitting no to the canonical FGtheta-theory but being certainly integrable à la Picard-Vessiot with the solvable Galois groups. One of good examples is the famous and fundamental Hermitian equation (containing a parameter) very closely related to the theory of Eq. (1).

Section 10 contains some conclusive remarks. 


\section{Background}

2.1. Commuting operators. The standard soliton/FG-solutions of integrable equations are known to be defined through the associated linear PDEs for the auxiliary $\Psi$-function:

$$
\widehat{\boldsymbol{L}}\left(\{U\} ; \partial_{x}\right) \Psi=\lambda \Psi, \quad \Psi_{t}=\widehat{\boldsymbol{A}}\left(\{U\} ; \partial_{x}\right) \Psi,
$$

where $\widehat{\boldsymbol{L}}$ and $\widehat{\boldsymbol{A}}$ are the ordinary differential (scalar or matrix) operators with coefficients $\{U\}$ being, in general, some functions of variables $(x, t):\{U\}:=\left\{u_{k}(x, t)\right\}$. The set of function $\{U\}$ is usually termed as the potential. This is because the first of Eqs. (2) contains no $\partial_{t}$ and thereby may be considered as a spectral problem defined by the operator expression $\widehat{\boldsymbol{L}}$. Moreover, the classical (spectral [30]) property of the potential $\{U\}$ to be finite-gap is determined by the spectrum of this eigenvalue problem and does not depend on $t$. On the other hand, the classical Burchnall-Chaundy-Baker (algebro-geometric) formulation [16, 7] uses one more spectral problem instead of second equation in (2):

$$
\widehat{\boldsymbol{L}}\left(\{U\} ; \partial_{x}\right) \Psi=\lambda \Psi, \quad \widehat{\boldsymbol{A}}\left(\{U\} ; \partial_{x}\right) \Psi=\mu \Psi,
$$

In both the formulations the nontrivial theory appears if equations (2) or (3) are compatible; there exists a common solution $\Psi$ and the potential is subjected to certain conditions. These are the well-known commutativities of operators $\left[\widehat{\boldsymbol{L}}, \partial_{t}-\right.$ $\widehat{\boldsymbol{A}}]=0$ for (2) and $[\widehat{\boldsymbol{L}}, \widehat{\boldsymbol{A}}]=0$ for (3).

2.1.1. Why one should pass from (2) to (3)? There is a simple explanation as to this question. Let the operator $\widehat{A}$ be determined by an hierarchy of some integrable PDEs

$$
U_{t}=\mathrm{K}([U]),
$$

where, as usual in a formal differential calculus [27] and in the sequel, the symbol $[U]$ is used to denote the finite set of derivatives $\left\{U, U_{x}, U_{x x}, \ldots\right\}$. Such hierarchies have been well tabulated in the literature [19, 28]. Regard Eqs. (2) from the viewpoint of their explicit integration (in some sense of the word). In a straightforward statement this problem is impossible to solve because $t$ is a hidden variable in the first of Eqs. (2). In fact, this variable may be thought of as an additional spectral one ${ }^{4}$ and the $t$-dependence of the potential $\{U\}$ is unknown/undetermined. Complexity of the question is not reduced until Eqs. (2) remain partial differential equations. Indeed, these equations do not have a general solution expressible in terms of any known functions. This is because Eqs. (4), integrable as they are, are not solvable in general. The only way, in order to solve the question, is to consider some particular situations when PDEs (4) admit transformations into some ODEs. Clearly, such a possibility is related to the self-similar reductions of Eqs. (4) and the most simple case is of course the reduction to the stationary variable $z=x-c t$. Assuming now the dependence $U=U(x-c t)$, we get, instead of (2),

$$
\widehat{\boldsymbol{L}}\left(U(z) ; \partial_{z}\right) \Psi=\lambda \Psi, \quad \Psi_{t}=\widehat{\boldsymbol{A}}\left(U(z) ; \partial_{z}\right) \Psi .
$$

The time $t$ disappears in the first of these equations and therefore there exists a solution in form of separability of variables: $\Psi=T(t) \cdot \psi(z)$. Substituting this

\footnotetext{
${ }^{4}$ This is so indeed because any additional parameter in coefficients of $\widehat{\boldsymbol{L}}\left(\{U\} ; \partial_{x}\right)$ may be formally viewed as a spectral variable defining a spectral operator pencil.
} 
ansatz into the last equations, we get immediately a separability parameter ${ }^{5} \mu$ and an exponential dependence $T=\exp (\mu t)$. Equations (2) thus acquire form (3):

$$
\widehat{\boldsymbol{L}}\left(\{U\} ; \partial_{z}\right) \psi=\lambda \psi, \quad\left(\widehat{\boldsymbol{A}}\left(\{U\} ; \partial_{z}\right)+c \partial_{z}\right) \psi=\mu \psi,
$$

where $U=U(z)$. Compatibility conditions of these equations $\left[\widehat{\boldsymbol{L}}, \widehat{\boldsymbol{A}}+c \partial_{z}\right]=0$ generate the stationary Lax-Novikov equations $F([U])=0[28]$ and, incidentally, different kind reductions may lead to other kind equations. For example, the general form of Painlevé equations and their ' $(\widehat{\boldsymbol{L}}, \widehat{\boldsymbol{A}})$-pairs' can be obtained by this way [47].

2.1.2. Algebraic curve and integrals of Lax-Novikov equations. Let $n, m$ be the orders of the operators $\widehat{\boldsymbol{L}}, \widehat{\boldsymbol{A}}$ respectively. Assuming that Eqs. (3) are compatible, we conclude, by elimination of the $\Psi$ from (3), that parameters $\lambda$ and $\mu$ are related by a polynomial dependence $W(\stackrel{n}{\mu}, \stackrel{m}{\lambda})=0$ and commuting operators $\widehat{\boldsymbol{L}}, \widehat{\boldsymbol{A}}$ themselves are also tied by the same dependence: $W(\widehat{\boldsymbol{A}}, \widehat{\boldsymbol{L}})$ is a zero operator $[16,7]$. The identity $W(\widehat{\boldsymbol{A}}, \widehat{\boldsymbol{L}})=0$ implies that its coefficients, being the differential functions $E_{j}$ of $\{U\}$, must be free constants $C_{j}$ independent of $x$. These yields a set of (compatible) ODEs $E_{j}([U])=C_{j}$ providing some integrals of motion $E_{j}$ for the Lax-Novikov equations mentioned above [19]. The further theory requires that the potential $\{U\}$ be the complex analytic function of the complex argument $x$.

2.2. Theta-function formulae. Solution to Eqs. (3) is an $n$-valued function of $\lambda$ (and an $m$-valued function of $\mu$ ) and this multi-valuedness is related to the algebraic equation $W(\mu, \lambda)=0$. This equation, being viewed as an algebraic curve over $\mathbb{C}$, defines a compact Riemann surface $\mathcal{R}$ of a finite genus. It is well known that multi-dimensional $\Theta$-functions are the universal tool in order to impart a 'single-valued form' to the analytic apparatus on Riemann surfaces of multi-valued algebraic functions [6]. Baker [7], initiated by work [16], was the first to transfer this $\lambda$-multi-valuedness of the $\Psi$ into a single-valued function of a point $\mathcal{P} \in \mathcal{R}$ and to construct the function itself through Riemann's $\Theta$-functions; the very first sentence of the work [7] indicates this. Akhiezer [3] arrived at the same objects when considering the problem (1) from completely different-purely spectral-viewpoint. In the 1970s all these discoveries were substantially developed, generalized [29, $30,41,23,37,38,25]$, and acquired their current $\Theta$-function form. The recent excellent survey by Matveev [43] is, perhaps, the best work both on the history of the question and background material. Most general and modern treatment of these results goes back to works by Krichever and reads as follows.

The spectral variable is thought of as the meromorphic function $\lambda=\lambda(\mathcal{P})$ in the sense that the complex number $\lambda$ in problem (2) is replaced with an abstract coordinate on $\mathcal{R}$ : the point $\mathcal{P}$. The variable $x$ is viewed as a parameter now and the function $\Psi$, as function on $\mathcal{R}$, is the function $\Psi(\mathcal{P})$ of an exponential type $[3,7]$ with essential singularities of some prescribed form [41, 37]. The formula realization of this result is known presently as a concept of the Baker-Akhiezer (BA) function [8]. The operator $\widehat{\boldsymbol{L}}$ as above and its coefficients (the potential $\{U\}$ ) is called the finite-gap or algebro-geometric. Of course, we could equally well say the same about $\mu=\mu(\mathcal{P})$ for operator $\widehat{\boldsymbol{A}}$ in problem (3).

\footnotetext{
${ }^{5}$ An independent treatment of the second eigenvalue of the second operator.
} 
The structure of solutions is universal $[7,37]$. For example, the scalar problem

$$
\widehat{\boldsymbol{L}} \Psi:=\frac{d^{n}}{d x^{n}} \Psi+u_{2}(x) \frac{d^{n-2}}{d x^{n-2}} \Psi+\cdots+u_{n}(x) \Psi=\lambda \Psi,
$$

and therefore equation (1), in the class of FG-potentials, has a solution which is given, under some normalization, by a general formula:

$$
\Psi(x ; \lambda(\mathcal{P}))=\frac{\Theta(x \boldsymbol{U}+\boldsymbol{D}+\mathfrak{U}(\mathcal{P}))}{\Theta(x \boldsymbol{U}+\boldsymbol{D})} \mathrm{e}^{\mathbf{I}(\mathcal{P}) x} .
$$

Distinctions between different FG-potentials $\left\{u_{k}(x)\right\}$ are only in the changes of the associated algebraic curve $W(\mu, \lambda)=0$ and the curve itself (its $\mathcal{R}$ with a canonical homology base $(\mathfrak{a}, \mathfrak{b}))$ determines all the quantities appearing in (6). Namely: $\Theta(\boldsymbol{z})$ is the canonical $g$-dimensional theta-series

$$
\Theta(\boldsymbol{z}):=\Theta(\boldsymbol{z} \mid \boldsymbol{\Pi})=\sum_{\boldsymbol{N} \in \mathbb{Z}^{g}} \mathrm{e}^{\pi \mathrm{i}\langle\boldsymbol{\Pi} \boldsymbol{N}, \boldsymbol{N}\rangle+2 \pi \mathrm{i}\langle\boldsymbol{N}, \boldsymbol{z}\rangle}
$$

of $g$ arguments $\boldsymbol{z}=\left(z_{1}, \ldots, z_{g}\right)$, built by an $\mathfrak{a}$-periods $\Pi$-matrix of normalized holomorphic Abelian integrals $\mathfrak{U}(\mathcal{P})=\left(\mathfrak{U}_{1}(\mathcal{P}), \ldots, \mathfrak{U}_{g}(\mathcal{P})\right)$ on $\mathcal{R}$; symbols like $\langle\boldsymbol{N}, \boldsymbol{z}\rangle$ denote Euclidian scalar product $\langle\boldsymbol{N}, \boldsymbol{z}\rangle:=N_{j} z_{j} ; \mathcal{P}$ is a free point on $\mathcal{R}$; $\Pi(\mathcal{P})$ is a normalized Abelian integral of the second kind with only pole of the first order at a point $\mathcal{P}_{\infty}$ at which $\lambda\left(\mathcal{P}_{\infty}\right)=\infty$; the vector $\boldsymbol{U}$ times $2 \pi \mathrm{i}$ is a vector of $\mathfrak{b}$-periods of this integral; $\boldsymbol{D}$ is an arbitrary constant $g$-vector. All this terminology is exhaustively expounded in the numerous literature (see, e.g., [6, 8, 18, 24, 25, $28,41])$ and the algebraic dependence $W(\mu, \lambda)=0$ is referred frequently to as the spectral curve. As for the problem (1), this curve constitutes a hyperelliptic equation of the form

$$
\mu^{2}=\left(\lambda-E_{1}\right) \cdots\left(\lambda-E_{2 g+1}\right)
$$

and all the FG-potentials are given by the famous Its-Matveev formula [41, 29]

$$
u(x)=-2 \frac{d^{2}}{d x^{2}} \ln \Theta(x \boldsymbol{U}+\boldsymbol{D})+\text { const } .
$$

Example 1. Most simple and popular example is a 1-gap potential. It is unique and is determined by the Weierstrass elliptic curve

$$
\mu^{2}=4(\lambda-e)\left(\lambda-e^{\prime}\right)\left(\lambda-e^{\prime \prime}\right) ;
$$

we denote its modulus as $\Pi=\omega^{\prime} / \omega$, where $\omega, \omega^{\prime}$ are Weierstrassian half-periods normalized by the condition $\Im \Pi>0$ [4]. Since this case is a 1-dimensional one, we replace $\mathcal{P} \mapsto \mathfrak{u}$ and $\operatorname{put}^{6} \mathfrak{U}(\mathcal{P})=\mathfrak{u}$. Therefore, normalizing integral $\mathbb{I}$, we have

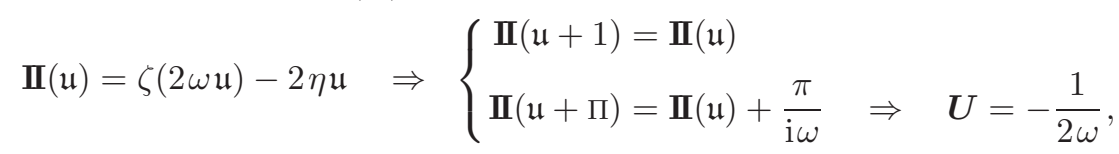

where $\zeta(z):=\zeta\left(z \mid \omega, \omega^{\prime}\right)$ and $\eta:=\eta\left(\omega, \omega^{\prime}\right)$ are the standard objects accompanying the theory of Weierstrassian function $\wp(z):=\wp\left(z \mid \omega, \omega^{\prime}\right)$ [4, 55]. Putting for simplicity $\boldsymbol{D}=0$, formulae (6) and (9) become

$$
u(x)=-2 \frac{d^{2}}{d x^{2}} \ln \Theta\left(\frac{x}{2 \omega} \mid \Pi\right)-2 \frac{\eta}{\omega}, \quad \Psi(x ; \lambda)=\frac{\Theta\left(\frac{x}{2 \omega}-\mathfrak{u} \mid \Pi\right)}{\Theta\left(\frac{x}{2 \omega} \mid \Pi\right)} \mathrm{e}^{\{\zeta(2 \omega \mathfrak{u})-2 \eta \mathfrak{u}\} x}
$$

\footnotetext{
${ }^{6}$ Jacobian of an elliptic curve is isomorphic to the curve itself.
} 
and $\lambda=\wp(2 \omega \mathfrak{u})$ (this is the formula $\lambda=\lambda(\mathcal{P})$ above). This potential is a precise equivalent of the classical Lamé form $u(x)=2 \wp\left(x-\omega-\omega^{\prime}\right)$ and 1-dimensional $\Theta(z \mid \Pi)$-series here coincides exactly with the Jacobi function $\theta_{3}(z \mid \Pi)$ defined by the standard formula (31).

All the constructions mentioned above-spectral, algebro-geometric, $\Theta$-function, and their varieties - are completely equivalent [38], which is why we shall refer to these approaches merely as spectral for short.

\section{Integrability of equation (1) by Picard-Vessiot}

3.1. The function $R$. The theory of equation (1) is closely related to the fundamental linear differential equation

$$
R_{x x x}-4(u+\lambda) R_{x}-2 u_{x} R=0
$$

determining function $R=R(x ; \lambda)$. Integrating this equation and denoting an integration constant as $\mu$, we get

$$
\mu^{2}=-\frac{1}{2} R R_{x x}+\frac{1}{4} R_{x}^{2}+(u+\lambda) R^{2}
$$

(see work [13] for a successive derivation of these formulae with use of Lie's symmetries approach.) Then the FG-solutions to the $\Psi$-function for Eq. (1), in general position $\mu \neq 0$, are given by the well-known formula

$$
\begin{aligned}
\Psi^{ \pm}(x ; \lambda) & =\sqrt{R(x ; \lambda)} \exp \int^{x} \frac{ \pm \mu d x}{R(x ; \lambda)} \\
& =\exp \int^{x} \frac{R_{x}(x ; \lambda) \pm 2 \mu}{2 R(x ; \lambda)} d x .
\end{aligned}
$$

Formulae (11)-(13) and their variations have been repeatedly appeared in the literature $[23,25,5,8,28]$. Their precise meaning, however, lies in the fact that the availability of formula (13) itself does not mean any integrability [13]. This is just an ansatz for Eq. (1) and its solution should be written down in terms of indefinite integrals; otherwise all the procedure would reduce to re-notations.

3.2. General formula for the $\Psi$-function. In the language of commuting Burchnall-Chaundy operators [16] an explicit formula for the $\Psi$-function (including (13)) results from the sequential elimination of derivatives $\Psi^{(k)}$ from the pair of differential equations (3) down to the formula

$$
\Psi_{x}=G([U] ; \lambda, \mu) \Psi \quad \Rightarrow \quad \Psi=\exp \int^{x} G([U] ; \lambda, \mu) d x .
$$

This simple recipe of getting the $\Psi$-function, perhaps, has no received mention in the modern literature [54]; it is, however, implicitly exploited in monograph [28]. Elimination of the last derivative $\Psi_{x}$ leads to equation of the curve $W(\mu, \lambda)=0$. The algebraic dependence $W(\widehat{\boldsymbol{A}}, \widehat{\boldsymbol{L}})=0$ serves, in some of works, as the basis for a formal definition of the algebraic integrability [38]. 
3.3. Novikov's equations and differential field. Motivated by the desire to define a differential field over which the integration is performed, we need to know the differential structure of function $R(x ; \lambda)$. It is well known that this function is a series in $\lambda$ with coefficients being differential polynomials in $u(x)$. Computational formulae for these polynomials have been detailed in the famous work [27, formulae (8)]. Finite order differential conditions on the potential appear if equation (11) has a solution being a polynomial in $\lambda$ [13]. As in the FG-theory, the equation (11) is also known in differential Galois theory as the second symmetric power of operator (1) [50, §4.3.4], [52, p. 671].

Definition 3.1. The potential $u(x)$ is said to be an FG-potential if equation (11) has a solution $R(x ; \lambda)$ being a polynomial in $\lambda$. No restrictions on coefficients of the curve $(12) \Leftrightarrow(8)$ have been imposed.

This definition is not a standard one but it is of course equivalent to the spectral [30, Theorem 1], algebraic [27, Ch. 3], or quadrature [21, 13] definitions. The only exception is a formal $\Theta$-function (algebro-geometric) setting because it does not use spectra, resolvent $R$, or quadratures. An important point here is the fact that the function domain for the potential is not defined as usual in Galois theory but calculated. This calculation is a problem of nonlinear integration, that is integration of ODEs for the potential $u(x)$. These are the famous Novikov equations [48]. We shall call this base field the Novikov differential field $\mathcal{N}([u])$ in $u(x)$-representation bearing in mind that $u(x)$ satisfies a Novikov equation $F\left(u, u_{x}, u_{x x}, \ldots, u_{x}^{(2 g+1)}\right)=0$. The field $\mathcal{N}([u])$ consists of rational functions of $u(x)$ over $\mathbb{C}(\lambda)$ and its derivatives. Parameter $\lambda$, the $g$ constants $c_{k}$ coming from the integral Gel'fand-Dickey recurrence [27], and branch points $E_{k}$ of the curve (12) belong to a subfield of constants for $\mathcal{N}([u])$. Here is an example of Novikov's equation under $g=2$ :

$$
\left(u_{x x x x x}-10 u u_{x x x}-20 u_{x} u_{x x}+30 u^{2} u_{x}\right)+c_{1}\left(u_{x x x}-6 u u_{x}\right)+c_{2} u_{x}=0 .
$$

Integration constant $\mu$, in an FG-class, is fixed to be dependent on the parameter in equation, that is $\mu=\mu(\lambda)$, and equation (12) turns into the formula (8). At the same time, as soon as $R(x ; \lambda)$ becomes a polynomial in $\lambda$ it becomes a differential polynomial $R([u] ; \lambda) \in \mathcal{N}([u])$. It should be noted that one suffices to have only one solution of Eq. (11) belonging to $\mathcal{N}([u]$ ) (see Sect. 9.1 further below).

3.4. Picard-Vessiot field, constants, and their hyperelliptic extension. As usual, the Picard-Vessiot extension

$$
\mathcal{N}([u])\left\langle\Psi^{ \pm}\right\rangle:=\mathcal{N}([u])\left(\Psi^{+}, \Psi^{-}, \Psi_{x}^{+}, \Psi_{x}^{-}\right),
$$

i. e., the splitting field, results from attaching to the field $\mathcal{N}([u])$ integrals $\Psi^{ \pm}$of equation (1) and its derivatives [32, 51, 34, 31, 45]. A simplest kind of extensions corresponds to solvable cases of the Galois theory and is known as the extension of Liouville [50, p. 33], [45, 31].

Definition 3.2. An extension $\widetilde{\mathcal{N}}$ of the differential field $\mathcal{N}$ is said to be Liouvillian if there exists a tower of fields $\mathcal{N}=\mathcal{N}_{0} \subset \mathcal{N}_{1} \subset \cdots \subset \mathcal{N}_{n}=\widetilde{\mathcal{N}}$ such that $\mathcal{N}_{k+1}=\mathcal{N}_{k}\left(\psi_{k}\right)$, where $\partial_{x} \psi_{k}$ or $\partial_{x} \ln \psi_{k}$ is an algebraic element over $\mathcal{N}_{k}$.

In other words, Liouvillian extensions are the natural enlargements of the base field performed by a step-by-step adjunction of solution to the simplest integrable ODEs: the 1st order linear ODEs

$$
\psi_{x}=A \psi+B
$$


with coefficients $(A, B)$ being algebraic/rational over previous step field. The standard adjunctions of an algebraic element $\boldsymbol{a}$, integral $\int p d x$, or exponential $\exp \int p d x$, where $p \in \mathcal{N}_{k}$, are obtained by putting here $(A, B)=\left(0, \boldsymbol{a}_{x}\right),(A, B)=(0, p)$, and $(A, B)=(p, 0)$ respectively.

Solvability of equation by quadratures is directly connected with a structure of the group constituting a differential version of the polynomial Galois group.

Definition 3.3. Differential Galois group $\operatorname{Gal}(\mathcal{N}\langle\Psi\rangle)$ of a linear ODE defined over $\mathcal{N}$ is a set of linear transformations of its solutions $\Psi$ 's that preserve all the algebraic (over $\mathcal{N}$ ) relations among $\Psi^{\prime}$ 's and their derivatives $\Psi^{(n)}$ (differential automorphisms group).

The Picard-Vessiot extension must have the same set of constants as $\mathcal{N}([u])$ [32], [34, p. 411], [50]. It is known that for equations of the form (1) the Wronskian $\Psi_{1}^{\prime} \Psi_{2}-\Psi_{2}^{\prime} \Psi_{1}$ is a constant. Taking (13) as one of possible bases for solutions, we get

$$
\begin{aligned}
\Psi_{x}^{+} \Psi^{-}-\Psi^{+} \Psi_{x}^{-} & =\sqrt{R_{x}^{2}-2 R R_{x x}+4(u+\lambda) R^{2}} \\
& =2 \mu(\lambda) .
\end{aligned}
$$

Clearly, it must be a constant of the Picard-Vessiot field whatever the potential $u(x)$ may be. Neglecting for the moment other constants, we obtain that field of constants $\mathbb{C}(\lambda)$ requires adjunction of the constant $\mu: \mathbb{C}(\lambda) \mapsto \mathbb{C}(\lambda, \mu)$. Character of this extension is determined by the $\lambda$-dependence of the function $R$. It may be polynomial, rational, or essentially transcendental. The case of rational polynomial $R(\lambda)$ is possible only if its poles do not depend on $x$; for we should otherwise have the $\lambda$-dependent poles of the $\Psi$-function, which is impossible by virtue of structure of Eq. (1). Hence the function $R$ must be an entire function of $\lambda$. The Galois group does depend in general on parameters of equation but we are interested in the following cases:

- When the Lie-Kolchin integrability structure is the same for generic $\lambda$ ?

Therefore two kinds of theories do exist, according as the field $\mathbb{C}(\lambda, \mu)$ does not, or does, belong to a finite algebraic or infinite extension of $\mathbb{C}(\lambda)$. The latter fields are excessively general because there are huge varieties of entire transcendental functions (without any classification) and they do not produce any restrictions on potential. On the other hand, finite extensions are of fixed algebraic (necessarily hyperelliptic) character and calibrated by the only number, namely, by the $\lambda$-degree $g$ of the polynomial $R([u] ; \lambda)$. In this case infinite Gel'fand-Dickey recurrences terminate and equation (12) leads to a finite set of differential restrictions on $u(x)$ in form of differential polynomials $E_{j}([u])=C_{j}$. This gives in fact yet another independent motivation (à la Picard-Vessiot) for appearance/availability of a polynomial solution to Eq. (11). For brevity, we shall adopt however the previous shorter notation for the base field $\mathcal{N}([u])$ without explicit indication of its $\lambda, \mu$-dependence $\mathcal{N}([u] ; \lambda, \mu)$ or dependencies on other field constants $\mathcal{N}\left([u] ; \lambda, \mu, C_{j}, \ldots\right)$, where dots stand for remaining constants which arise upon complete integration of a Novikov equation.

3.5. Finite-gap Galois groups. Below is a characterization of the Galois group of equation (1) in the class of FG-potentials. Condition on parameter $\lambda$ of 
being an arbitrary quantity is a fundamental requirement meant throughout the paper.

Theorem 3.4. The Picard-Vessiot extension $\mathcal{N}([u])\left\langle\Psi^{ \pm}\right\rangle$is a Liouvillian extension of the transcendence degree equal to 1. Associated group $\operatorname{Gal}\left(\mathcal{N}([u])\left\langle\Psi^{ \pm}\right\rangle\right)$, under generic $\lambda \neq E_{j}$, is connected and isomorphic to the group $\mathfrak{G}=\left(\begin{array}{cc}\alpha & 0 \\ 0 & \alpha^{-1}\end{array}\right)$, where $\alpha \in \mathbb{C}$. For other values of $\lambda$ it is isomorphic to group $\mathfrak{G}=\left(\begin{array}{cc} \pm 1 & \alpha \\ 0 & \pm 1\end{array}\right)$.

Proof. For generic $\lambda$ 's integral (13) does not belong to $\mathcal{N}([u])$. From (13) it follows that this extension is Liouvillian. Take the canonical basis of solutions (13). Then the three quantities $\left\{\Psi^{-}, \Psi_{x}^{+}, \Psi_{x}^{-}\right\}$are expressed through the transcendent being adjoined $\Psi^{+}$as follows:

$$
\Psi^{-}=\frac{R}{\Psi^{+}}, \quad \Psi_{x}^{+}=\frac{R_{x}+2 \mu}{2 R} \Psi^{+}, \quad \Psi_{x}^{-}=\frac{R_{x}-2 \mu}{2 \Psi^{+}} \quad(\mu \neq 0) .
$$

Clearly, in the case of such $\lambda$ 's that $\mu=0$ these relations cease to be valid since $\left(\Psi^{+}, \Psi^{-}\right)$become linearly dependent on each other. The relations should be modified and we choose the following basis $\Psi^{ \pm}$:

$$
\left(\Psi^{-}\right)^{2}=R, \quad \Psi_{x}^{-}=\frac{R_{x}}{2 R} \Psi^{-}, \quad \Psi_{x}^{+} \Psi^{-}-\Psi^{+} \Psi_{x}^{-}=1 .
$$

In both of these cases we adjoin one transcendental element (respectively):

$$
\Psi^{+}=\exp \int^{x} \frac{R_{x}+2 \mu}{2 R} d x \quad \text { or } \quad \Psi^{+}=\sqrt{R} \int^{x} \frac{d x}{R} .
$$

In the latter case the radical $\sqrt{R}=\Psi^{-}$can sometimes be element of $\mathcal{N}([u])$ as an example of the Lamé equations shows [55, Ch. 23], [40].

Let us check invariance of relations (18)-(19) with respect to linear transformation of the basis

$$
\left(\begin{array}{c}
\Psi^{+} \\
\Psi^{-}
\end{array}\right) \mapsto\left(\begin{array}{c}
\alpha \Psi^{+}+\beta \Psi^{-} \\
\gamma \Psi^{+}+\delta \Psi^{-}
\end{array}\right)
$$

this determines the Galois group $\mathfrak{G}=\left(\begin{array}{ll}\alpha & \beta \\ \gamma & \delta\end{array}\right)$ completely. In case (18) we get the following set of equalities:

$$
\alpha \gamma=0, \quad \beta \delta=0, \quad \alpha \delta+\beta \gamma=1, \quad \beta=0 .
$$

From this it follows that $\beta=0, \gamma=0$, and $\alpha \delta=1$. The number $\alpha$ can not be any algebraic one; for we should otherwise have a finite Galois group and the algebraic $\Psi^{ \pm}$-solutions to give a contradiction with a single-valuedness of the general formula (6). In case (19) we derive that $\delta^{2}=1, \gamma=0, \alpha \delta=1$, and no conditions on $\beta$ (except for degenerate cases of curve). Hence in generic case $\lambda \neq E_{j}$ the group $\mathfrak{G}$ is connected. Its possible forms are thus as follows:

$$
\mathfrak{G}=\left(\begin{array}{cc}
\alpha & 0 \\
0 & \alpha^{-1}
\end{array}\right) \quad \text { or } \quad \mathfrak{G}=\left(\begin{array}{cc}
\delta & \beta \\
0 & \delta^{-1}
\end{array}\right),
$$

where $\delta= \pm 1$ (compare with cases 3, 4 in Proposition 2.2 of [45]).

To summarize briefly, we conclude that independently of the topological genus of the curve (8), 'finite-gap' groups $\operatorname{Gal}\left(\mathcal{N}([u])\left\langle\Psi^{ \pm}\right\rangle\right)$do not depend on parameter $\lambda$ and cease to be diagonal and connected only for isolated values of the parameter. 
Corollary 3.5. Equations (1) of the FG-class are factorizable over $\mathcal{N}([u])$ :

$$
\partial_{x x}-(u+\lambda)=\left(\partial_{x}+\frac{1}{2} \frac{R_{x}}{R} \pm \frac{\mu}{R}\right)\left(\partial_{x}-\frac{1}{2} \frac{R_{x}}{R} \mp \frac{\mu}{R}\right) .
$$

REMARK 1 . We used nowhere any specific form of the polynomial $R$. Theorem 3.4 is easily restated for arbitrary integrable $\lambda$-pencils of the 2 nd order with the only condition that $R(x ; \lambda) \in \mathcal{N}([u])$. Recall that the spectral $\lambda$-pencil is a generalization of the canonical spectral equation of the form (5) to more complex (e.g. polynomial) dependencies of the differential expression $\widehat{\boldsymbol{L}}$ on the external parameter $\lambda$, that is $\widehat{\boldsymbol{L}}\left(\{U\} ; \partial_{x}, \lambda\right)=0$. An example is the well-known spectral $\lambda$-pencil of the form

$$
\Psi_{x x}-\frac{u_{x}}{u} \Psi_{x}-\left(\lambda^{2}-\frac{u_{x}}{u} \lambda+u v\right) \Psi=0 ;
$$

it arises when integrating the integrable nonlinear Schrödinger equation [28, 46, 8]. In Sect. 9 we shall consider other examples of the $\lambda$-pencils (see also [13, Sect. 5(a)]).

By virtue of structure of the formula (14) Theorem 3.4 has a direct generalization.

THEOREM 3.6. Let $\mathcal{N}([U])$ be a Novikov field associated with the pair of Burchnall-Chaundy scalar operators (3). Then both of these equations are integrable in Liouvillian extensions of the same transcendence degree. Under generic $\lambda, \mu$ the differential Galois groups of these equations are connected and isomorphic to the diagonal groups $\mathfrak{G}=\operatorname{Diag}(\alpha, \beta, \ldots, \gamma) \subset \mathrm{GL}(\mathbb{C})$.

Novikov's equations are known to be Hamiltonian systems integrable by Liouville [19]. However such a way of their integration is not necessary since determination of the potential, i. e., construction of the field $\mathcal{N}([U])$, is given by formulae following completely from the 'linear' Picard-Vessiot theory. It does not require Hamiltonians.

We finish this section with a digression to one remarkable example. It is a Matveev 1-positon potential given by the seemingly elementary formula [42]

$$
u=-2 \ln _{x x}\{\sin (a x+b)-a x-c\} .
$$

Surprisingly, in spite of its complete fitting into the integration scheme above, it is not amenable to integration by means of any classical algorithm in the PicardVessiot theory (Kovacic [35], Singer [51]). Indeed, these algorithms are applicable to the finite algebraic extensions of $\mathbb{C}(x)$, whereas this $u \in \mathbb{C}\left(x, \mathrm{e}^{\mathrm{i}(a x+b)}, a, c\right)$.

\section{Spectral/quadrature duality. An integration procedure}

4.1. Drach-Dubrovin equations. The quadrature Drach approach gives a very simple explanation as to why and where the fundamental polynomial

$$
R([u] ; \lambda)=\left(\lambda-\gamma_{1}(x)\right) \cdots\left(\lambda-\gamma_{g}(x)\right)
$$

comes from, what its roots $\gamma_{k}$ are, and why these are precisely the quantities that complete the indefinite quadratures ${ }^{7}$. In what concerns the spectral viewpoint, a remarkable result by Dubrovin $[22,23]$ is that the quantities $\gamma_{k}$ arise as zeroes of a $\Theta$-function since they solve the inversion problem of Jacobi [3, 41, 30].

\footnotetext{
${ }^{7}$ An elementary explanation to appearance of these objects (supplemented with Russian translation of works [20, 21]) can be found in [12].
} 
Lemma 4.1 (Drach [21], Dubrovin [22]). Functions $\gamma_{k}(x)$ satisfy the system of $O D E s$

$$
\frac{d \gamma_{k}}{d x}=2 \frac{\sqrt{\left(\gamma_{k}-E_{1}\right) \cdots\left(\gamma_{k}-E_{2 g+1}\right)}}{\prod_{j \neq k}\left(\gamma_{k}-\gamma_{j}\right)}, \quad j, k=1, \ldots, g
$$

and potential is determined by the trace formula [29]

$$
u=2 \sum_{k=1}^{g} \gamma_{k}(x)-\sum_{k=1}^{2 g+1} E_{k} .
$$

Proof. One inserts (22) into (12) and takes (8) into account. Collecting the result in degrees $\left(\lambda-\gamma_{k}\right)^{n}$, one requires identity under arbitrary $\lambda$. One gets (23) and (24).

REMARK 2. We presented such a way of proof because it is applicable to higher order operators and even spectral $\lambda$-pencils. The reason is that the derivation of trace formulae is not evident when generalizing. Formulae of such a kind cease actually to be the 'trace formulae' because they have no longer Gel'fand's treatment of the operator trace analogs. They are also not derivable directly from the definition of $R$-polynomial like (22); illustrative counterexamples can be found in work [11].

4.2. Weierstrass theorem and new representation for the $\Psi$-function. Main content of this section was briefly announced in [13] and we shall present here the extensive proofs, derivations, and precise correlation between spectral and quadrature approaches.

THEOREM 4.2. Let $u(x)$ be an FG-potential corresponding to the arbitrary curve (8). Then solution to equation (1) is given by the quadratures

$$
\Psi^{ \pm}(x ; \lambda)=\exp \frac{1}{2}\left\{\int^{\gamma_{1}(x)} \frac{w \pm \mu}{z-\lambda} \frac{d z}{w}+\cdots+\int^{\gamma_{g}(x)} \frac{w \pm \mu}{z-\lambda} \frac{d z}{w}\right\},
$$

where $w^{2}=\left(z-E_{1}\right) \cdots\left(z-E_{2 g+1}\right)$ and functions $\gamma_{k}=\gamma_{k}(x)$ are determined through inversion of the set of indefinite integrals

$$
\sum_{k=1}^{g} \int^{\gamma_{k}} z^{g-1} \frac{d z}{w}=2 x+a_{g}, \quad \sum_{k=1}^{g} \int^{\gamma_{k}} z^{n} \frac{d z}{w}=a_{n+1}, \quad n=0,1, \ldots, g-2 .
$$

The FG-potential u(x) is determined by formula (24).

Proof. Let us substitute (22) into (13) and change the integration variable $x$ to $z$. We then obtain the following rules

$$
\begin{array}{ll}
\frac{R_{x}}{R} d x=d \ln \prod_{k}\left(\lambda-\gamma_{k}\right) & \rightarrow \frac{1}{z-\lambda} d z, \\
\frac{2 \mu}{R} d x=\frac{2 \mu}{\prod_{j}\left(\lambda-\gamma_{j}\right)} \cdot \frac{1}{2} \frac{d \gamma_{k}}{\varrho_{k}} \prod_{j \neq k}\left(\gamma_{k}-\gamma_{j}\right) & \rightarrow \frac{-\mu}{z-\lambda} \frac{d z}{w},
\end{array}
$$

wherein $\varrho_{k}^{2}=\left(\gamma_{k}-E_{1}\right) \cdots\left(\gamma_{k}-E_{2 g+1}\right)$. Abelian integrals of 3rd kind, as appeared in (25), result from differential equations (23). Furthermore, substitution of expression 
(25) into equation (1) leads, to get an identity, to formula (24); this can serve as yet another way of derivation of the trace formula. Symmetrizing right hand sides of equations (23), we rewrite them down in a form that admits an application of the indefinite integration operations, that is (26). This set determines $\gamma_{k}$ as functions of $x$.

CoROLlary 4.3. In $u(x)$-representation the extension $\mathcal{N}([u])\left\langle\Psi^{ \pm}\right\rangle$requires the one quadrature (13). If formula (25) is used then extensions $\mathcal{N}([u])\left\langle\Psi^{ \pm}\right\rangle$consist in an adjunction of a symmetric sum of the logarithmic Abelian integrals.

Remark 3 (Definition). Special attention must be given to the fact that integrability of the 'linear $\Psi$ ' is also algebraic (hyperelliptic) as is nonlinear integrability of $\mathcal{N}([u])$. More precisely, in what follows the term 'algebraic' will mean that ultimate answers contain finitely many indefinite integrals of algebraic functions and inversions of the formers.

As for transition from primary $\lambda$-dependence to the $x$-one and vice versa, the duality between spectral and quadrature representations is nontrivial; it is characterized by the following statement.

THEOREM 4.4. Quadrature and spectral approaches are equivalent in the sense that the explicit transition between formula (25) and its spectral counterpart (see below) is realized through the Weierstrass theorem on permutation of limits and parameters in a normalized Abelian integral of third kind.

Proof. Sub-exponential expression in (25) is a sum of Abelian integrals, each with logarithmic singularities on $\mathcal{R}$ at two points: $(z, w)=(\lambda,+\mu)$ and branch place $(z, w)=(\infty, \infty)$. According to a Weierstrass theorem, we may exchange singularities of an elementary logarithmic integral with its limits $[6,18,41]$. In our case, these are $(z, w)=(\gamma(x), \varrho(x))$ and a lower limit $(z, w)=(\alpha,+\beta)$, where $\beta^{2}=\left(\alpha-E_{1}\right) \cdots\left(\alpha-E_{2 g+1}\right)$. More precisely, switching the places

$$
\left\{\begin{array}{c}
(\gamma(x), \varrho(x)) \\
(\alpha, \beta)
\end{array}\right\} \rightleftarrows\left\{\begin{array}{c}
(\lambda, \mu) \\
(\infty, \infty)
\end{array}\right\},
$$

we obtain that the difference

$$
\int_{\alpha}^{\gamma(x)} \frac{w+\mu}{z-\lambda} \frac{d z}{w}-\int_{\infty}^{\lambda}\left\{\frac{w+\varrho(x)}{z-\gamma(x)}-\frac{w+\beta}{z-\alpha}\right\} \frac{d z}{w}=\cdots
$$

is to be everywhere finite quantity, that is certain holomorphic integral:

$$
\cdots=\int^{\gamma(x)}\left\{A_{1}(\lambda)+A_{2}(\lambda) z+\cdots+A_{g}(\lambda) z^{g-1}\right\} \frac{d z}{w} .
$$

Sum of $g$ such quantities must be a holomorphic integral depending symmetrically on $\gamma$ 's. Expression (25) is thus converted to its dual object

$$
\Psi^{ \pm} \simeq \exp \frac{1}{2}\left\{\int^{\lambda} \frac{w \pm \varrho_{1}(x)}{z-\gamma_{1}(x)} \frac{d z}{w}+\cdots+\int^{\lambda} \frac{w \pm \varrho_{g}(x)}{z-\gamma_{g}(x)} \frac{d z}{w}+\operatorname{holomorhic}(\lambda, x)\right\}
$$


This is nothing else but the spectral formula by Its \& Matveev [30] deserving to be mentioned more often. We reproduce ${ }^{8}$ their result as it has been written in [30, p. 351]:

$$
\omega(\lambda)=\int_{\beta_{n}}^{\lambda} \frac{M(\lambda)}{2 \sqrt{p(\lambda)}} d \lambda, \quad \int_{\beta_{j-1}}^{\alpha_{j}} d \omega(\lambda)=0, \quad j=1, \ldots, n,
$$

where $M(\lambda)=\lambda^{n}+a_{1} \lambda^{n-1}+\ldots+a_{n}$ and

$$
\begin{gathered}
\omega_{k}(\lambda)=\int_{\infty}^{\lambda}\left(\frac{\sqrt{P(\lambda)}+\sqrt{P\left(\lambda_{k}(x)\right)}}{\lambda-\lambda_{k}(x)}-\frac{\sqrt{P(\lambda)}+\sqrt{P\left(\lambda_{k}(0)\right)}}{\lambda-\lambda_{k}(0)}+M_{k}(\lambda)\right) \frac{d \lambda}{2 \sqrt{P(\lambda)}}, \\
\psi(x, \lambda)=\exp \left(\mathrm{i} x \omega(\lambda)+\sum_{k=1}^{n} \omega_{k}(\lambda)\right) .
\end{gathered}
$$

Meaning of all the quantities presented in (28)-(30) and transition (25) $\rightleftarrows(30)$ are obvious from the context. To put it differently, the permutation theorem of Weierstrass, regarding inverse transition $(30) \rightarrow(25)$, is a way of doing normalization of periods of these integrals so that all the parameters in the spectral formulae (28)-(30) can be 'dumped' to a common multiplication constant for the $\Psi$. By this means the non-indefinite integrals (27) or (29) with a parametrical dependence on transcendental functions $\gamma_{k}(x)$ turn into the indefinite ones (25) of an algebraic function.

Description of invariant property of equation (1) to be integrable has been completed and we conclude the section with the comments about fundamental difference between spectral and quadrature modes of getting the formulae.

4.3. On a Riemann surface. At this point not only do analysis on Riemann surfaces does not come into play, but also the surfaces themselves do not appear. One has just a designation

$$
\mu:=\sqrt{\left(\lambda-E_{1}\right) \cdots\left(\lambda-E_{2 g+1}\right)}
$$

and the theory consists of elementary substitutions (see footnote on p. 3). On the other hand, verification of spectral formulae (28)-(30) is a highly nontrivial task since they contain a complete set of transcendental objects of Riemann's theory of Abelian integrals and differentiation of an integrand containing $\gamma$ 's. In this respect, not using the permutation theorem, the 'spectral integral' in (27), that is

$$
\int_{\infty}^{\lambda} \frac{w \pm \varrho(x)}{z-\gamma(x)} \frac{d z}{w}
$$

would be very akin to an integral representation of any special function, say, complete elliptic Legendre's integral

$$
K(x)=\int_{0}^{1} \frac{d z}{\sqrt{\left(1-z^{2}\right)\left(1-x^{2} z^{2}\right)}} .
$$

${ }^{8}$ We have not found mention of this important result in the literature. See also formula (3) in Akhiezer's work [3]. 
YU. BREZHNEV

The latter is not expressible by means of any finite Liouvillian extension over $\mathbb{C}(x)$ since $K\left(x^{2}\right)$ satisfies a 2 nd order irreducible ${ }_{2} F_{1}\left(\frac{1}{2}, \frac{1}{2} ; 1 \mid x^{2}\right)$-hypergeometric equation [4].

REMARK 4 (history). Both the integrabilities are due to Liouville but chronologically, 'linear integrability' (1830-40s) was preceded by more famous nonlinear integrability of Hamiltonian systems (1840-50s). Despite the numerous modern literature, the fact that these two kinds of integrability are non-casually related to the one name Liouville was first observed by Morales-Ruiz [45, pp. 51-52].

\section{The $\Theta$-functons}

By virtue of the fact that extension $\mathcal{N}([u])\left\langle\Psi^{ \pm}\right\rangle$is transcendental, analytic representations for the previous formulae require introducing new functions. These are the $\Theta$-series (7) involved to the theory by Matveev and Its in their famous work [30].

TheOREM 5.1. The $\Theta$-function representations (6), (9) are deducible from quadrature (25). The expressions (6) and (25) are proportional to each other.

Proof of this theorem and consecutive derivation of formula (6) from (25) have been detailed in $[13, \S 7]$. An important point here is the deductive appearance of all the aggregates of formula (6) when it is viewed as an axiomatic one: the integral $\Pi(\mathcal{P})$, its periods $\boldsymbol{U}$, and the Abel map $\mathfrak{U}(\mathcal{P})$.

From this theorem, we may draw the conclusion that when recognizing the integrability of linear equations the $\Theta$-functions themselves are not necessary. They realize a step to be considered as the next one after emergence of an integral symbol $\int$ in (13) and (26).

5.1. Some differential properties of theta-functions. Theorem $5.1 \mathrm{sug}$ gests a search for representation of the field $\mathcal{N}([u])$ by means of $\Theta$-functions. To do this require some differential properties of $\Theta$-functions and we show further that they are available. All the FG-theory tells us that Abelian and BA-functions satisfy certain differential identities. By these identities are meant the fact that Abelian functions, as theta-function ratios of linear sections of $g$-dimensional jacobians of curves, have a lot of differential relations between themselves and many of such relations have forms of known integrable PDEs [15, 28]. Adding here exponential functions of the BA-type, we involve into analysis $(\widehat{\boldsymbol{L}}, \widehat{\boldsymbol{A}})$-pairs for these PDEs. Moreover, let FG-potential be expressible through the $\theta$-functions of Jacobi. Then not only do Abelian and BA-functions satisfy certain differential identities but $\theta$ functions themselves also satisfy some ODEs.

Denote by $\theta[\varepsilon]$ the standard $\theta$-series of Jacobi with characteristics $(\varepsilon, \delta)[4]$ :

$$
\theta[\delta](x \mid \tau):=\sum_{-\infty}^{\infty} \mathrm{e}^{\pi \mathrm{i}\left(k+\frac{\varepsilon}{2}\right)^{2} \tau+2 \pi \mathrm{i}\left(k+\frac{\varepsilon}{2}\right)\left(z+\frac{\delta}{2}\right)}
$$

i. e., $\theta\left[\begin{array}{l}1 \\ 1\end{array}\right]=-\theta_{1}, \theta\left[\begin{array}{l}1 \\ 0\end{array}\right]=\theta_{2}, \theta\left[\begin{array}{l}0 \\ 0\end{array}\right]=\theta_{3}, \theta\left[\begin{array}{l}0 \\ 1\end{array}\right]=\theta_{4}$. Let $\vartheta:=\theta(0 \mid \tau)$ be corresponding $\vartheta$-constants and $\theta_{1}^{\prime}(x \mid \tau)$ stands for $x$-derivative of the series $-\theta\left[\begin{array}{l}11 \\ 1\end{array}(x \mid \tau)\right.$. Period of the meromorphic elliptic integral is denoted by $\eta=\zeta(1 \mid 1, \tau)$. 
THEOREM 5.2. Jacobian functions $\theta[\varepsilon], \theta_{1}^{\prime}$ with arbitrary integral characteristics are differentially closed over the field of the $\left(\vartheta^{2}, \eta\right)$-constants and satisfy the autonomous ODEs

$$
\left\{\begin{array}{c}
\frac{\partial \theta\left[\begin{array}{c}
\varepsilon \\
\delta
\end{array}\right]}{\partial x}=\frac{\theta_{1}^{\prime}}{\theta\left[\begin{array}{l}
1 \\
1
\end{array}\right]} \theta\left[\begin{array}{l}
\varepsilon \\
\delta
\end{array}\right]-(-1)^{\left[\frac{\delta}{2}\right] \varepsilon} \pi \vartheta\left[\begin{array}{c}
\varepsilon \\
\delta
\end{array}\right]^{2} \cdot \frac{\theta\left[\begin{array}{c}
\varepsilon-1 \\
0
\end{array}\right] \theta\left[\begin{array}{c}
0 \\
\delta-1
\end{array}\right]}{\theta\left[\begin{array}{l}
1 \\
1
\end{array}\right]} \\
\frac{\partial \theta_{1}^{\prime}}{\partial x}=\frac{\theta_{1}^{\prime 2}}{\theta\left[\begin{array}{l}
1 \\
1
\end{array}\right]}-\pi^{2} \vartheta\left[\begin{array}{l}
0 \\
0
\end{array}\right]^{2} \vartheta\left[\begin{array}{l}
0 \\
1
\end{array}\right]^{2} \cdot \frac{\theta\left[\begin{array}{l}
1 \\
0
\end{array}\right]^{2}}{\theta\left[\begin{array}{l}
1 \\
1
\end{array}\right]}-4\left\{\eta+\frac{\pi^{2}}{12}\left(\vartheta\left[\begin{array}{c}
0 \\
0
\end{array}\right]^{4}+\vartheta\left[\begin{array}{c}
0 \\
1
\end{array}\right]^{4}\right)\right\} \cdot \theta\left[\begin{array}{c}
1 \\
1
\end{array}\right]
\end{array},\right.
$$

where $\left[\frac{\delta}{2}\right]$ signifies an integer part of the number $\delta / 2$.

These formulae are consequences of more general differential properties of Jacobi's functions briefly tabulated in [14]. One can see that the similar properties are inherent characteristics of the general $\Theta$-functions if the $g$-dimensional jacobian is isomorphic to a product of elliptic curves. Many examples of such reductions can be found in [8].

Example 2. Define a $\Theta$-function with characteristics $\left[\begin{array}{c}\boldsymbol{\alpha} \\ \boldsymbol{\beta}\end{array}\right]$ as follows:

$$
\Theta\left[\begin{array}{c}
\boldsymbol{\alpha} \\
\boldsymbol{\beta}
\end{array}\right](\boldsymbol{z} \mid \Pi):=\mathrm{i}^{\langle\boldsymbol{\alpha}, \boldsymbol{\beta}\rangle} \Theta\left(\boldsymbol{z}+\frac{1}{2} \Pi \boldsymbol{\alpha}+\frac{1}{2} \boldsymbol{\beta} \mid \Pi\right) \cdot \mathrm{e}^{\pi \mathrm{i}\left\langle\boldsymbol{\alpha}, \boldsymbol{z}+\frac{1}{4} \Pi \boldsymbol{\alpha}\right\rangle} .
$$

Then in the case $g=2$ we have the following identity.

Proposition 5.3. The reduction formula for genus $g=2$ under $\Pi_{12}=\frac{1}{2}$ :

$$
\begin{aligned}
& \Theta\left[\begin{array}{c|cc}
\alpha \varepsilon \\
\beta \delta
\end{array}\right]\left(\begin{array}{ccc}
\frac{1}{2} z-\frac{1}{8} \alpha \tau & \frac{1}{4} \tau & \frac{1}{2} \\
w-\frac{1}{4} \alpha & \frac{1}{2} & \varkappa
\end{array}\right)= \\
& =\mathrm{e}^{\frac{\pi}{2} \mathrm{i} \alpha\left(z+\beta+\frac{1}{4} \alpha \tau\right)} \cdot\left\{\theta\left[\begin{array}{l}
0 \\
\varepsilon
\end{array}\right](z \mid \tau) \theta\left[\begin{array}{l}
\varepsilon \\
\delta
\end{array}\right](w \mid \varkappa)+\mathrm{i}^{2 \beta+\varepsilon} \cdot \theta\left[\begin{array}{l}
1 \\
\varepsilon
\end{array}\right](z \mid \tau) \theta\left[\begin{array}{c}
\varepsilon \\
\delta \\
\delta
\end{array}\right](w \mid \varkappa)\right\} .
\end{aligned}
$$

Differential properties of these $\Theta, \Theta^{\prime}$-functions follow completely from Theorem 5.2.

This example is a rather illustrative one because curves have often symmetries and if a genus- 2 curve has an involutory symmetry differed from the hyperelliptic one $(\lambda, \mu) \mapsto(\lambda,-\mu)$ then one can show that its $\Pi$-matrix is reducible to form (34). Formula (34) is perhaps a simplest case of reduction of the theta-functions to the two elliptic tori $\tau$ and $\varkappa$. More complex equivalent of (34) is presented in [8].

COROllary 5.4. Let jacobian of the curve (8) be splittable into a product of the elliptic curves and the collective symbols $\theta, \vartheta, \eta$ stand for arising Jacobi's theta-functions and their constants. Then the field $\mathbb{C}_{\partial}\left(\theta ; \vartheta^{2}, \eta, \ldots\right)$ is a differential extension of $\mathcal{N}([u])$ (dots indicate other constants of the field).

Example 3. Non-elliptic 2-gap potential (9) with $\Theta=\Theta\left[\begin{array}{l}00 \\ 00\end{array}\right]$ for the reduction case (34):

$$
u=-2 \ln _{x x}\left\{\theta_{4}(U x+A \mid \tau) \theta_{2}(V x+B \mid \varkappa)-\mathrm{i} \theta_{1}(U x+A \mid \tau) \theta_{1}(V x+B \mid \varkappa)\right\},
$$

where $\{\tau, \varkappa, A, B, V\}$ are arbitrary. Since the differential $\theta$-calculus is completely at hand, we get a particular but nontrivial example of solution to Dubrovin's effectivization formulae for genus $g=2$. Recall that the problem consists [24] in determination of the 'winding' vector $\boldsymbol{U}$ and is described by a system of equations containing the undetermined fourth derivatives of the $\Theta$-function [24]. In the example under consideration the ultimate answer turns out to be quite finite but somewhat large to display here. Equation for the one sought-for quantity $U$ is an algebraic equation of degree 9 (exercise: derive it). 
5.2. Linearly exponential divisor and $\Theta$-representation of $\mathcal{N}([u])$. Let us use notation of formula (6) and plug into (9) an inessential exponential multiplier:

$$
u(x)=-2 \frac{d^{2}}{d x^{2}} \ln \Theta(x \boldsymbol{U}+\boldsymbol{D}) \mathrm{e}^{h x}+\text { const } .
$$

Introduction of this term is motivated by the fact that solutions for the $\Psi$-function (6) are expressed not only through the $\Theta$-functions but involve an exponential factor. We shall call the quantity $\Theta(x \boldsymbol{U}+\boldsymbol{D}) \mathrm{e}^{h x}$, with $h, \boldsymbol{U}$, and $\boldsymbol{D}$ being constants with respect to $\partial$, the linearly exponential divisor or $\mathfrak{L}_{x}$-divisor. Let us form a field $\mathbb{C}\left(\mathfrak{L}_{x}\right)$. The following proposition gives a weaker property than Corollary 5.4, but it is valid for arbitrary genera.

Proposition 5.5. The field $\mathbb{C}\left(\mathfrak{L}_{x}\right)$ is $\partial$-differential and finitely generated.

Proof. Expression (36) satisfies a Novikov equation which has finite order $2 g+1$. Hence the derivatives $\frac{d^{n}}{d x^{n}} \Theta(x \boldsymbol{U}+\boldsymbol{D}) \mathrm{e}^{h x}$ of order $n \geqslant(2 g+1)+2$ are expressed rationally through $\mathfrak{L}_{x}$ and its lower derivatives.

The field $\Theta_{\partial}=\mathbb{C}_{\partial}(\Theta(x \boldsymbol{U}+\boldsymbol{D}))$ may be considered as a field generated by one linear divisor $(h=0)$. It is obviously that $\mathcal{N}([u]) \subset \Theta_{\partial}$. Of course, $\Theta_{\partial}$ contains now not only Abelian functions but this extension is well defined since $\Theta$-series and $\mathfrak{b}$-periods $\boldsymbol{U}$ are computed once $u(x)$ has been given. For this reason, we can redefine equation (1) as one given over $\Theta_{\partial}$ and thereby we let

$$
\mathcal{N}(\Theta):=\mathbb{C}_{\partial}(\Theta(x \boldsymbol{U}+\boldsymbol{D})) .
$$

Although $\mathcal{N}([u]) \varsubsetneqq \mathcal{N}(\Theta)$, the field $\mathcal{N}(\Theta)$ is said to be a $\Theta$-representation of Novikov's fields. The constant $\lambda$, for the moment, may be disregarded.

It should be emphasized that in the FG-integration there appears not merely an abstract multi-dimensional $\Theta$-series but its specification $\mathfrak{L}_{x}$; the 1-dimensional linear section of jacobians. Moreover, the most general $\Theta$-function is an object defined up to an exponential multiplier (see (33)) so that we may think of it, and therefore of the divisor $\mathfrak{L}_{x}$, as a continual generalization of $\Theta$-function with discrete characteristics. Thus, Liouvillian solutions $\mathcal{N}([u])\left\langle\Psi^{ \pm}\right\rangle$are expressed through the $\Theta$ with a linear dependence of its arguments upon $x$ and the 'linearly exponential' multiplier ${ }^{9} \mathrm{e}^{\mathbf{I I}(\mathcal{P}) x}$ first explicitly appeared in Akhiezer's work [3] and subsequently was axiomatized by Krichever [37].

REMARK 5. In what follows we shall exhibit that the continually parametric object $\mathfrak{L}_{x}$ may be introduced in its own right, in a particular case, through the quadrature integrable ODEs. Interestingly, the different kind sections of theta-function arguments can lead to other important equations. One remarkable property of such a kind appears even in the $g=1$ case. Let us consider the function $\theta(x \mid \tau)$ as a function on a simplest (i. e., straight line) section of the 2-dimensional variety $\{$ jacobian $\otimes$ moduli space $\}$. Without loss of generality we may impart to this function the form $\theta_{1}(A \tau+B \mid \tau)$. Then this object generates the general Hitchin class of solutions to the sixth Painlevé equation in a form exactly as does the finite-gap formula (9), that is logarithmic derivative of a ratio of entire functions [14].

\footnotetext{
${ }^{9}$ This 'linear exponent' is a result of the contemporary theory; Baker [7] did not specify an exponential $\Theta$-structure of solutions but just cited to pp. 275, 289 of his [6].
} 


\section{Integration as a linearly exponential $\Theta$-extension}

In this section we give a formulation of the $\Theta$-function scheme as integration à la Picard-Vessiot. Let us consider the above Picard-Vessiot extension [50] $\mathcal{N}([u]) \subset \mathcal{N}([u])\left\langle\Psi^{ \pm}\right\rangle$in the representation $u(x)$. This transcendence is Liouvillian and dependence of the $\Psi$ on parameter $\lambda$ is also transcendental contrary to the 'rationality' of the field $\mathbb{C}(\lambda, \mu)$. Meanwhile, based on Theorems 3.4 and 5.1, we see that the field has the following structure:

$$
\mathcal{N}([u])\left\langle\Psi^{ \pm}\right\rangle=\mathbb{C}_{\partial}\left(\frac{\Theta(x \boldsymbol{U}+\boldsymbol{D}+\mathfrak{U}(\mathcal{P}))}{\Theta(x \boldsymbol{U}+\boldsymbol{D})} \mathrm{e}^{\mathbf{I}(\mathcal{P}) x}\right) .
$$

It immediately follows that if we pass to the $\Theta$-representation (37) then integration procedure can be reduced to one operation. Namely, a field, over which an equation has been defined, is supplemented with an element of the same form as one generating the field itself:

$$
\mathcal{N}([u]) \subset \mathcal{N}(\Theta) \subset \mathbb{C}_{\partial}\left(\Theta(x \boldsymbol{U}+\boldsymbol{D}) \mathrm{e}^{h x}, \Theta(x \boldsymbol{U}+\boldsymbol{D}+\mathfrak{U}(\mathcal{P})) \mathrm{e}^{\mathbf{I}(\mathcal{P}) x}\right) .
$$

It is significant in this viewpoint that problem of the 'linear integration' drops out along with the problem of building the base field $\mathcal{N}([u])$ being treated as a problem of the nonlinear integration. In the $\Theta$-representation the potential is determined only by means of operations in the field $\mathcal{N}(\Theta)$; formula (36).

THeOREM 6.1. All the embeddings

$$
\mathcal{N}([u]) \subset \mathcal{N}(\Theta) \subset \mathcal{N}([u])\left\langle\Psi^{ \pm}\right\rangle \subset \mathbb{C}_{\partial}\left(\Theta(x \boldsymbol{U}+\boldsymbol{D}), \Theta(x \boldsymbol{U}+\boldsymbol{D}+\mathfrak{U}(\mathcal{P})) \mathrm{e}^{\mathbb{I}(\mathcal{P}) x}\right)
$$

are the Liouvillian extensions.

Proof. The fact that embedding $\mathcal{N}([u]) \subset \mathcal{N}(\Theta)$ is Liouvillian follows directly form formula (9). The statement concerning the second embedding is a consequence of (13) because $R([u] ; \lambda) \in \mathcal{N}(\Theta)$. Rewriting formula (6) in the form

$$
\Theta(x \boldsymbol{U}+\boldsymbol{D}+\mathfrak{U}(\mathcal{P})) \mathrm{e}^{\mathbf{I}(\mathcal{P}) x}=\Psi^{+} \cdot \Theta(x \boldsymbol{U}+\boldsymbol{D}),
$$

we deduce that property for the last embedding to be Liouvillian results from the proportionality of (6) and expression (13) for $\Psi^{+}$(Theorems 4.2 and 5.1).

Theorem 6.2. For generic $\lambda$ integration of equation (1) in the $\Theta$-representation is equivalent to a multiplication of an element $\Xi(x)$ generating the field $\mathcal{N}(\Theta)$ by an adjoined linearly exponential divisor:

Proof. Consider $\Xi(x)=\Theta(x \boldsymbol{U}+\boldsymbol{D})^{-1}$. It is clear that $\mathbb{C}_{\partial}(\Xi)=\mathcal{N}(\Theta)$. Then

$$
\Psi^{ \pm}(x ; \lambda(\mathcal{P}))=C_{ \pm} \cdot \Xi(x) \cdot \Theta(x \boldsymbol{U}+\boldsymbol{D} \pm \mathfrak{U}(\mathcal{P})) \mathrm{e}^{ \pm \mathbf{I}(\mathcal{P}) x}
$$

because all the holomorphic/meromorphic integrals on hyperelliptic curves change sign under permutation of sheets $\mu \mapsto-\mu$; we may write $\pm \mathfrak{U}(\mathcal{P}), \pm \mathbf{I}(\mathcal{P})$ in (39).

This theorem has an important treatment:

- When passing to the $\Theta$-representation the equation (1) is integrated as if it were an equation with constant coefficients. Integration procedure is thus trivialized under a proper choice of 'domain of rationality'. The inverse transform method for the soliton class is a particular case of this general construction. 
Indeed, the simplest FG-case corresponds to the 0-gap one with $\mathcal{N}(\Theta)=\mathbb{C}(\lambda)$ and we need only one linear exponent; the construction (39) acquires the form

$$
\Psi^{ \pm}(x ; \lambda)=C_{ \pm} \cdot \Xi(x) \cdot \mathrm{e}^{ \pm a(\lambda) x}, \quad \Xi(x)=\mathrm{e}^{0 \cdot x} \in \mathcal{N}(\Theta),
$$

wherein $\Xi(x)$ has the 'same form' as the adjoint element $\mathrm{e}^{a(\lambda) x}$. Adjoining all the exponents associated with an $N$-soliton solution (and their varieties like positons (21) or rational solitons), we obtain the general 0-gap case.

REMARK 6. The structure of solution (39) in form of simple multiplication of elements generating $\mathcal{N}(\Theta)$ and its extension $\mathcal{N}([u])\left\langle\Psi^{ \pm}\right\rangle$is not quite typical for equations integrable by attaching the linear exponents [35] or, especially, for equations with a solvable Galois group [32]. This property owes its origin to the availability of $\lambda$ in equation (Theorem 3.4).

Transition between $u$ - and $\Theta$-representations is transcendental with respect to $\lambda$-dependence and other constants of the field. These constants are the $\Pi$-matrices of curves, $\boldsymbol{U}$-periods, and vector $\boldsymbol{D}$. We may therefore trivialize the scheme above if we proceed further and redefine equation (1) over (37) as one given over the $\lambda$-pencil (field) of the $\mathfrak{L}_{x}(\mathcal{P})$-divisors:

$$
\mathfrak{L}_{x}(\mathcal{P}):=\Theta(x \boldsymbol{U}+\boldsymbol{D}+\mathfrak{U}(\mathcal{P})) \mathrm{e}^{\mathbf{I}(\mathcal{P}) x} .
$$

Although field $\mathbb{C}_{\partial}\left(\mathfrak{L}_{x}(\mathcal{P})\right)$ is generated by the infinite number of elements, equation (1) itself constitutes an infinite $\lambda$-pencil of equations. Strictly speaking, both spectral and quadrature approaches require to look upon Eq. (1) as being a differentiallyalgebraic one: differential in $x$ and algebraic (polynomial) in $\lambda$. Furthermore, by virtue of Proposition 5.5, the arbitrary $\mathfrak{L}_{x}(\mathcal{P})$-divisor generates some solution of Novikov's equation. It may be fixed by choice of one element of the pencil $\mathfrak{L}_{x}(\mathcal{P})$ :

$$
\mathcal{N}(\Theta) \rightarrow \mathbb{C}_{\partial}\left(\Theta\left(x \boldsymbol{U}+\boldsymbol{D}_{0}+\mathfrak{U}\left(\mathcal{P}_{0}\right)\right) \mathrm{e}^{\mathbb{I I}\left(\mathcal{P}_{0}\right) x}\right) .
$$

Having extended the field (41), that is $\mathbb{C}_{\partial}\left(\mathfrak{L}_{x}\left(\mathcal{P}_{0}\right)\right)$, to the field $\mathbb{C}_{\partial}\left(\mathfrak{L}_{x}(\mathcal{P})\right)$, its Galois group becomes trivial since the integral of equation (1) is given now in form of a ratio of two field elements.

\section{Integrability and differential closedness}

7.1. Differential closure in terms of $\theta$-functions. Attaching the divisor $\mathfrak{L}_{x}(\mathcal{P})$ as transcendental element with a parameter $\mathcal{P}$ tells us that it should be introduced to the theory as the base function, along with the available $\Theta$ 's without parameter $\mathcal{P}$. Owing to Theorem 6.2 this would arrive us at a closed differential apparatus (differential closedness) accompanying spectral equation and potential. Presently, the general $\Theta$-formula realization of this viewpoint is an open problem, which is why we illustrate it by cases when $g$-dimensional $\Theta$-function reduces to a combination of Jacobian ones.

At first glance, from Theorem 5.2, it would seem that supplement of the basis (32) with $\mathfrak{L}_{x}$-divisor of the type $\theta_{1}(x-\mathfrak{u}) \mathrm{e}^{h x}$ requires also adjunction of all the functions $\theta_{1}^{\prime}, \theta_{2,3,4}(x-\mathfrak{u})$. That no such complication takes place will be apparent from the following statement. 
Theorem 7.1. For Weierstrassian curve (10) with modulus $\tau=\omega^{\prime} / \omega$ one defines an elliptic $\mathfrak{L}_{x}(\mathcal{P})$-divisor $\Lambda$ by the formula

$$
\Lambda(x ; \mathfrak{u} \mid \tau):=\theta_{1}(x-\mathfrak{u} \mid \tau) \exp \left(\frac{\theta_{1}^{\prime}(\mathfrak{u} \mid \tau)}{\theta_{1}(\mathfrak{u} \mid \tau)} x+h x\right), \quad \mathfrak{u} \notin \mathbb{Z} \tau+\mathbb{Z},
$$

where $h$ is an extra parameter. Then the six functions $\Lambda, \theta_{1}^{\prime}$, and $\theta_{k}(k=1,2,3,4)$ satisfy the closed autonomous system of ODEs over the field $\mathbb{C}\left(\eta, \vartheta^{2}, \theta(\mathfrak{u})\right)$ :

$$
\left\{\begin{aligned}
\frac{\partial \theta_{k}}{\partial x} & =\frac{\theta_{1}^{\prime}}{\theta_{1}} \theta_{k}-\pi \vartheta_{k}^{2} \cdot \frac{\theta_{n} \theta_{m}}{\theta_{1}}, \quad n=\frac{8 k-28}{3 k-10}, \quad m=\frac{10 k-28}{3 k-8} \\
\frac{\partial \theta_{1}^{\prime}}{\partial x} & =\frac{\theta_{1}^{\prime 2}}{\theta_{1}}-\pi^{2} \vartheta_{3}^{2} \vartheta_{4}^{2} \cdot \frac{\theta_{2}^{2}}{\theta_{1}}-4\left\{\eta+\frac{\pi^{2}}{12}\left(\vartheta_{3}^{4}+\vartheta_{4}^{4}\right)\right\} \cdot \theta_{1} \\
\frac{1}{\Lambda} \frac{\partial \Lambda}{\partial x} & =\frac{\theta_{1}^{\prime}}{\theta_{1}}+\frac{\pi \vartheta_{2}^{2}}{\theta_{1}(\mathfrak{u})} \cdot \frac{\theta_{1}^{3}(\mathfrak{u}) \cdot \theta_{2} \theta_{3} \theta_{4}+\theta_{2}(\mathfrak{u}) \theta_{3}(\mathfrak{u}) \theta_{4}(\mathfrak{u}) \cdot \theta_{1}^{3}}{\theta_{1} \cdot\left(\theta_{2}^{2}(\mathfrak{u}) \cdot \theta_{1}^{2}-\theta_{1}^{2}(\mathfrak{u}) \cdot \theta_{2}^{2}\right)}+h
\end{aligned}\right.
$$

Motivation for the theorem lies in the fact that Abelian integrals, on the one hand, are representable in terms of theta-functions and, on the other hand, are differentially closed: the base integrals of 1st, $2 \mathrm{nd}$, and 3rd kind form a differential basis. Indeed, derivatives of integrals are functions and any function is expressed through the two base ones $\left(\wp, \wp^{\prime}\right)$ (generators of an elliptic functions field) which are in turn integrals of exact meromorphic differentials of 2 nd kind. However thetafunction representation for a 3rd kind integral is still lacking in system (32).

Proof. Meromorphic functions are at hand since they are formed by $\theta$-quotients. Derivatives of meromorphic functions are again meromorphic ones but differentiation of $\theta_{k}(x)$ generates $\theta_{1}^{\prime}(x)$. The quotient $\theta_{1}^{\prime} / \theta_{1}$ is proportional to the Weierstrass $\zeta$-function which in turn represents a meromorphic (i. e., 2nd kind) elliptic integral [4]; it is alone as genus $g=1$. Without loss of generality we may set that the missing integral of 3rd kind has two logarithmic singularities. The place of one of them may be fixed at $x=0$ and the second place can be taken as a parameter. Call it $\mathfrak{u}$. Residues of a corresponding differential are opposite in sign and they can be moved to a common multiplication constant. Such an integral III is unique (even for arbitrary $g$ ) up to a holomorphic one(s) since all the other integrals are expressed through $\mathbf{I I I}=\mathbf{I I}(x ; \mathfrak{u})$. Its derivatives are again meromorphic functions and the process closes. It will suffice to add an exponent of $\mathbf{I I}$ and integral $\mathbf{I I}$ itself is given by the known formula

$$
\operatorname{III}(x ; \mathfrak{u}):=\frac{1}{2} \int^{z} \frac{w+w_{0}}{z-z_{0}} \frac{d z}{w}=\ln \frac{\sigma(2 x-2 \mathfrak{u})}{\sigma(2 x)} \mathrm{e}^{2 \zeta(2 \mathfrak{u}) x},
$$

where $z=\wp(2 x), w=\wp^{\prime}(2 x)$ and $z_{0}=\wp(2 \mathfrak{u}), w_{0}=\wp^{\prime}(2 \mathfrak{u})$. Weierstrassian parameters $\left(\omega, \omega^{\prime}\right)$, presented in (43), are replaced by the one quantity $\tau$ thanks to homogeneity relation $\omega^{2} \wp\left(\omega x \mid \omega, \omega^{\prime}\right)=\wp(x \mid 1, \tau)=: \wp(x \mid \tau)$. Holomorphic integral is absent in system (32) but is present in a basis of Abelian integrals. Missing element $\Lambda_{0}$ can be formally adjoined by setting $\Lambda_{0}(x \mid \tau)=x$ and supplementing system (42) with equation $\frac{d \Lambda_{0}}{d x}=1$. Adding to (43) the holomorphic integral $h \Lambda_{0}$ and converting the right hand side of (43) to the $\theta$-functions, one obtains that adjunction of $\exp ($ III $)$ is equivalent to adjunction of the object $\Lambda(x ; \mathfrak{u} \mid \tau)$. Differentiating (43) and converting it to the $\theta$ 's, one arrives, after some simplification, at the last equality in Eqs. (42). 
If $\mathfrak{u} \in \mathbb{Z} \tau+\mathbb{Z}$ then the integral III turns into a logarithm of meromorphic function: $\ln (\wp(x)-e)$. There is nothing to adjoin.

Corollary 7.2. Let potential be a finite-gap one and g-dimensional jacobian split into a product of the elliptic curves. Then differentiation of $g$-dimensional functions $\Theta(x \boldsymbol{U}+\boldsymbol{D})$ reduces to a set of the '1-dimensional' equations (42). The functions $\theta_{k}, \theta_{1}^{\prime}$ and $\Lambda$, taken possibly with different moduli $\tau$, form the differentially closed basis over which every Novikov's equation of order $\leqslant 2 g+1$ and problem (1) are integrated.

In the framework of this corollary integrability of Novikov's equations is a manifestation of differential closedness of the first two equations in (42). The third equation in (42) 'integrates' equations with a parameter and their consequences (see examples in [13]). Here, 'integrates' means that all these equations are nothing more than combinations of system (42) and its derivatives. Let us consider some examples.

Example 4. The two gap Lamé potential

$$
\Psi_{x x}=\{24 \wp(2 x \mid \tau)+\lambda\} \Psi .
$$

This is a classical example presented in many places [8, 28]. Corresponding solution expressed in terms of our objects reads as follows:

$$
\begin{gathered}
\Psi^{ \pm}=\frac{d}{d x} \frac{\Lambda(x ; \pm \mathfrak{u} \mid \tau)}{\theta_{1}(x \mid \tau)}, \quad h=\frac{ \pm 2 \mu}{3 \lambda^{2}-12^{2} g_{2}(\tau)}, \quad \wp(2 \mathfrak{u} \mid \tau)=\frac{\lambda^{3}+12^{3} g_{3}(\tau)}{36 \lambda^{2}-12^{3} g_{2}(\tau)}, \\
\mu^{2}=\left(\lambda^{2}-48 g_{2}(\tau)\right)\left(\lambda^{3}-36 g_{2}(\tau) \lambda+432 g_{3}(\tau)\right) \quad(\Leftrightarrow(8)),
\end{gathered}
$$

where $g_{2}(\tau), g_{3}(\tau)$ are the standard modular $\tau$-representations for Weierstrassian parameters $a, b$ entering into the curve $w^{2}=4 z^{3}-a z-b$ [4]. Novikov's equation (15) is satisfied under $\left(c_{1}, c_{2}\right)=\left(0,-672 g_{2}(\tau)\right)$ and $R$-polynomial has the form

$$
R([u] ; \lambda)=\lambda^{2}-\frac{1}{2} u \lambda+\frac{1}{4} u^{2}-36 g_{2}(\tau),
$$

where $u=24 \wp(2 x \mid \tau)$.

7.2. Non-elliptic example. We consider here a potential being no elliptic function but expressible through the 'elliptic' $\theta$-functions.

Example 5. The $\Psi$-function for non-elliptic potential (35). It is a nonlinear superposition of the one-gap $\Psi$-functions. If we denote for brevity $z=U x+A$ and $w=V x+B$ we then derive that

$$
\Psi(x ; \lambda)=\frac{\Lambda\left(z+\frac{1}{2} \tau ; \mathfrak{U}_{1} \mid \tau\right) \Lambda\left(w+\frac{1}{2} ; \mathfrak{U}_{2} \mid \varkappa\right)-\Lambda\left(z ; \mathfrak{U}_{1} \mid \tau\right) \Lambda\left(w ; \mathfrak{U}_{2} \mid \varkappa\right)}{\theta_{1}\left(z+\frac{1}{2} \tau \mid \tau\right) \theta_{1}\left(w+\frac{1}{2} \mid \varkappa\right)-\theta_{1}(z \mid \tau) \theta_{1}(w \mid \varkappa)} \mathrm{e}^{\mathbf{I}(\lambda) x},
$$

where holomorphic integrals $\mathfrak{U}_{1}, \mathfrak{U}_{2}$ are the certain linear combinations $\mathfrak{U}_{k}=C_{k j} \mathfrak{u}_{j}$ of the elliptic holomorphic ones $\mathfrak{u}_{1}, \mathfrak{u}_{2}$ because the curve (8) corresponding to the $\Theta$-function (34) has the form

$$
\mu^{2}=\lambda(\lambda-1)(\lambda-\boldsymbol{a})(\lambda-\boldsymbol{b})(\lambda-\boldsymbol{a b})=: P_{5}(\lambda)
$$


and is realized as covers of two tori defined by moduli $\tau$ and $\varkappa$ :

$$
\begin{gathered}
\wp\left(2 \mathfrak{u}_{1} \mid \tau\right)+\vartheta_{2}^{4}(\tau)+\vartheta_{3}^{3}(\tau)=3 \frac{(1-\boldsymbol{a})(1-\boldsymbol{b}) \lambda}{(\lambda-\boldsymbol{a})(\lambda-\boldsymbol{b})} \vartheta_{3}^{4}(\tau), \\
\wp\left(2 \mathfrak{u}_{2} \mid \varkappa\right)+\vartheta_{2}^{4}(\varkappa)+\vartheta_{3}^{3}(\varkappa)=3 \frac{(1-\boldsymbol{a})(1-\boldsymbol{b}) \lambda}{(\lambda-\boldsymbol{a})(\lambda-\boldsymbol{b})} \vartheta_{3}^{4}(\varkappa) .
\end{gathered}
$$

These are the classical formulae by Jacobi [8] presented in terms of Weierstrass' $\wp$ and the pair of branch points $\{\boldsymbol{a}, \boldsymbol{b}\}$ and moduli $\{\tau, \varkappa\}$ are commonly written down for one another [8]. All the information concerning this curve can be found in [8] and we omit details of some calculations. We need to compute the integral $\mathbf{I}(\lambda)$.

Abelian integrals for the curve (46) are expressed through $\theta, \theta_{1}^{\prime}$ and therefore derivation of the meromorphic integral $\mathbf{I}(\lambda)$ is a routine calculation. An explanation is that the reduction of holomorphic integrals to elliptic ones entails the reduction of the meromorphic Abelian integrals to the meromorphic elliptic ones. The latter are expressed through Weierstrassian $\zeta$-function, i. e., $\theta^{\prime} / \theta$, and meromorphic elliptic functions. First translate formulae for cover (47) into the language of $\theta$-functions:

$$
\frac{\vartheta_{2}^{2}(\tau)}{\vartheta_{3}^{2}(\tau)} \frac{\theta_{4}^{2}\left(\mathfrak{u}_{1} \mid \tau\right)}{\theta_{1}^{2}\left(\mathfrak{u}_{1} \mid \tau\right)}=\frac{(1-\boldsymbol{a})(1-\boldsymbol{b}) \lambda}{(\lambda-\boldsymbol{a})(\lambda-\boldsymbol{b})}, \quad \frac{\vartheta_{2}^{2}(\tau)}{\vartheta_{3}^{2}(\tau)} \frac{\theta_{4}^{2}\left(\mathfrak{u}_{1} \mid \tau\right)}{\theta_{1}^{2}\left(\mathfrak{u}_{1} \mid \tau\right)}=\frac{\vartheta_{2}^{2}(\varkappa)}{\vartheta_{3}^{2}(\varkappa)} \frac{\theta_{4}^{2}\left(\mathfrak{u}_{2} \mid \varkappa\right)}{\theta_{1}^{2}\left(\mathfrak{u}_{2} \mid \varkappa\right)} .
$$

This is a complete set of equations determining the holomorphic integrals $\mathfrak{u}_{1}, \mathfrak{u}_{2}$ as functions of $\lambda$. The point $\lambda=\infty$ corresponds to the values $\mathfrak{u}_{1}=\frac{1}{2} \tau$ and $\mathfrak{u}_{2}=\frac{1}{2} \varkappa$. Drop out for the moment indication of modulus $\tau$ in the following transformation of a meromorphic elliptic integral:

$$
\begin{aligned}
\int \frac{s d s}{\sqrt{4 s^{3}-g_{2} s-g_{3}}} & =\left|s=\frac{\pi^{2}}{12}\left(\vartheta_{3}^{4}+\vartheta_{4}^{4}-3 \vartheta_{3}^{2} \vartheta_{4}^{2} \frac{\theta_{3}^{2}\left(\mathfrak{u}_{1}\right)}{\theta_{4}^{2}\left(\mathfrak{u}_{1}\right)}\right)\right| \\
& =-\frac{1}{2} \frac{\theta_{1}^{\prime}\left(\mathfrak{u}_{1}-\frac{1}{2} \tau\right)}{\theta_{1}\left(\mathfrak{u}_{1}-\frac{1}{2} \tau\right)}-2 \eta \mathfrak{u}_{1}=\cdots
\end{aligned}
$$

On the other hand, the 1st formula in (48) supplemented with use of the standard quadratic $\vartheta, \theta$-identities brings this integral into the following expression:

$$
\cdots=\text { const } \int s \cdot \frac{\lambda-\sqrt{\boldsymbol{a}} \sqrt{\boldsymbol{b}}}{\sqrt{P_{5}(\lambda)}} d \lambda \simeq \int\left(\frac{(\lambda-\boldsymbol{a})(\lambda-\boldsymbol{b})}{(1-\boldsymbol{a})(1-\boldsymbol{b}) \lambda}+\frac{\vartheta_{2}^{4}+\vartheta_{3}^{4}}{3 \vartheta_{3}^{4}}\right) \frac{\lambda-\sqrt{\boldsymbol{a}} \sqrt{\boldsymbol{b}}}{\sqrt{P_{5}(\lambda)}} d \lambda,
$$

that is meromorphic integral on the curve (46) with a pole at $\lambda=\infty$ and a surplus one at $\lambda=0$. Doing the same for the second torus $\left(\mathfrak{u}_{2}\right)$ with modulus $\varkappa$, we obtain one more meromorphic integral with the same infinities. Forming their linear combination, we can construct the integral with a single singularity at infinite point. After some algebraic simplifications the sought-for result becomes:

$$
\begin{aligned}
\mathbf{I}(\lambda) & =a \cdot \frac{\theta_{1}^{\prime}\left(\mathfrak{u}_{1}-\frac{1}{2} \tau \mid \tau\right)}{\theta_{1}\left(\mathfrak{u}_{1}-\frac{1}{2} \tau \mid \tau\right)}+b \cdot \frac{\theta_{1}^{\prime}\left(\mathfrak{u}_{2}-\frac{1}{2} \varkappa \mid \varkappa\right)}{\theta_{1}\left(\mathfrak{u}_{2}-\frac{1}{2} \varkappa \mid \varkappa\right)}+c \cdot \mathfrak{u}_{1}+d \cdot \mathfrak{u}_{2} \\
& =a \cdot \frac{\theta_{1}^{\prime}\left(\mathfrak{u}_{1} \mid \tau\right)}{\theta_{1}\left(\mathfrak{u}_{1} \mid \tau\right)}+b \cdot \frac{\theta_{1}^{\prime}\left(\mathfrak{u}_{2} \mid \varkappa\right)}{\theta_{1}\left(\mathfrak{u}_{2} \mid \varkappa\right)}+\frac{(\lambda+p) \mu}{\lambda(\lambda-\boldsymbol{a})(\lambda-\boldsymbol{b})}+q \cdot \mathfrak{u}_{1}+r \cdot \mathfrak{u}_{2},
\end{aligned}
$$

where constants $\{a, b, c, d, p, q, r\}$ depend only on parameters of the potential (35), i. e., on $\{\tau, \varkappa, A, B, V\}$, and are independent of $\lambda$. Again, after careful co-ordination of all the moduli and normalizing constants the direct check of (1), (35), (45), and (49) becomes a good exercise in a differential $\theta$-calculus. We mention in passing 
that this example cannot be elaborated in the framework of the standard elliptic soliton theory [8].

7.3. A new treatment of the spectral parameter. In proof of Theorem 7.1 parameter $\mathfrak{u}$ was the only 'external' parameter independent of 'internal' parameters of the curve (moduli). On the other hand, the only parameter being external to the field $\mathcal{N}([u])$ and equation (1) is $\lambda$. It plays an isolated role. Apart from the fact that it is merely present in equation, it may be treated as an object arising from the two independent 'mechanisms': 1) adjunction of a transcendental element and, on the other hand, 2) differential closedness of all the Abelian integrals. As evidenced by the foregoing and formula (25), these are the same things:

- The logarithmic singularity in a canonical integral of third kind is arbitrary and independent of moduli. The property of the theory to be integrable is, by construction, independent of it. It may therefore always be thought of as a (free) spectral variable.

The converse is also true. Differentiation of the 3rd kind integrals yields other integrals and functions. In other words, informally speaking, one may say that

- Closed class of ODEs integrable through $\Theta(x \boldsymbol{U}+\boldsymbol{D})$ is in fact integrable in terms of $\left\{\Theta, \Theta^{\prime}, \mathfrak{L}_{x}\right\}$ and 'owes' to contain an external (except for moduli) parameter $\mathcal{P}$.

Indeed, there is one fundamental logarithmic integral III for each algebraic curve and it has a single parameter $\mathcal{P} \Leftrightarrow \lambda$. In the elliptic case this III has form (43) and for arbitrary genera it is expressed through the $g$-dimensional $\Theta$ 's [6]. The one fold logarithmic $\partial_{x}$-derivative of (25), i. e., derivatives of $\mathbf{I I}\left(\gamma^{\prime} \mathrm{s} ; \mathcal{P}\right)$, yields the rational functions of $(z, w)\left(\gamma^{\prime} \mathrm{s}\right)$ and therefore only meromorphic objects remain since integrals themselves disappear. As a rough guide we have here $\partial_{x} \exp (\mathbf{I I I})=\mathbf{I I I}_{x} \cdot \exp (\mathbf{I I I})$. This linear in $\exp (\mathbf{I I I})$ equation ${ }^{10}$ is treated as a spectral one. Coefficients of this equation (more precisely its $\lambda$-independent pieces) can be thought of as potential. The quantity $\mathcal{P}$ should always be distinguished as an external one because, otherwise, the following chain

$$
\text { external } \lambda \Leftrightarrow \partial_{x} \text {-closedness } \Leftrightarrow{ }^{\prime} \Psi \text {-linearity' }
$$

is destroyed altogether. Moreover, the $x$-dependence $\Theta(x \boldsymbol{U}+\boldsymbol{D})$ is not bound to be a linear one and spectral equations must not necessarily be of the form (3). Counterexamples in Sect. 9 illustrate these points.

\section{Definition of $\theta$ through Liouvillian extension}

Insomuch as the object $\Lambda(x ; \mathfrak{u} \mid \tau)$ is in fact a theta-function with a parameter, we can use the differential equations described above as the basis for a definition of the theta's themselves and, in particular, consider character of their integrability.

Proposition 8.1. System (32) has the two algebraic (rational) integrals

$$
\vartheta_{2}^{2} \theta_{4}^{2}-\vartheta_{4}^{2} \theta_{2}^{2}=A_{1} \vartheta_{3}^{2} \theta_{1}^{2}, \quad \vartheta_{2}^{2} \theta_{3}^{2}-\vartheta_{3}^{2} \theta_{2}^{2}=A_{2} \vartheta_{4}^{2} \theta_{1}^{2}
$$

generalizing the famous Jacobi $\theta$-identities when $A_{1} \neq 1 \neq A_{2}$.

\footnotetext{
${ }^{10} \mathrm{An}$ important remark is in order. We might not say the same as applied to the 'pure spectral' object (27) since non-indefinite integral remains. Again, Weierstrass' theorem does the job. The 'one-fold $\partial_{x}$ ', which is equivalent here to the ' $\partial_{x}$-differential closedness', explains why the spectral equations are always linear.
} 
PROOF. The straightforward calculation shows that $\partial_{x} A_{1}=\partial_{x} A_{2}=0$.

In a nutshell, the differential genesis of the object $\Lambda$ is as follows. Function $\theta_{1}^{\prime}$ is determined differentially through $\theta_{1}$. Therefore two functions with two arbitrary constants solve the system (32). One of constants serves a homogeneity $\theta \mapsto C \theta$ of (32). Another one $\mathfrak{u}$ is non-algebraic and is related to an autonomy of equations (32). Hence the two transcendental functions $\theta_{1}(x-\mathfrak{u} \mid \tau)$ and $\theta_{2}(x-\mathfrak{u} \mid \tau)$ remain. These functions are represented, up to a shift and holomorphic integral, by the one object $\Lambda(x ; \mathfrak{u} \mid \tau)$.

THEOREM 8.2. Differential equations (42) are algebraically integrable.

Proof. By a direct computation one can show that any solution $\theta_{k}=\theta$ of the system (42) satisfies the same 5th order ODE

(50) $\left(\frac{1}{F_{x}}\left(\frac{F_{x}^{2}}{F}\right)_{x}\right)_{x}+8 F_{x}=0, \quad F=(\ln \theta)_{x x}-2 \kappa, \quad-\kappa:=2 \eta+\frac{1}{6} \pi^{2}\left(\vartheta_{3}^{4}+\vartheta_{4}^{4}\right)$.

Therefore, not taking into account $\mathfrak{u}$, there is only one essential parameter in equations (42), i. e., parameter $\kappa$. From this it follows that

$$
F=\Xi(x ; \boldsymbol{a}, \boldsymbol{b}, \boldsymbol{c}): \quad \int^{F} \frac{d z}{\sqrt{z(z-\boldsymbol{a})(z-\boldsymbol{b})}}=2 \mathrm{i} x+\boldsymbol{c}
$$

and integration is thus completed if, according to definition in Remark 3, we adjoin the inversion operation $\Xi$ :

$$
\theta=\exp \int^{x}\left\{\int^{x} \Xi(y ; \boldsymbol{a}, \boldsymbol{b}, \boldsymbol{c}) d y\right\} d x \cdot \mathrm{e}^{\kappa x^{2}+\boldsymbol{d} x+\boldsymbol{e}}
$$

where $\boldsymbol{a}, \boldsymbol{b}, \boldsymbol{c}, \boldsymbol{d}, \boldsymbol{e}$ are the integration constants. Of course, the inversion function $\Xi$ here bears no relation to ratios of the $\theta$-series. Integration of equations for functions $\theta_{1}^{\prime}$ and $\Lambda$ is obvious.

REMARK 7. In a separate work we shall show that the algebraic integrability above can be supplemented with a Hamiltonian formulation $\dot{X}=\Omega \nabla \mathcal{H}(X)$ to the system (42) and its Lagrangian description.

It is noteworthy, that variable $\theta$ satisfies an equation of fifth order, not third, as it would be expected from the well-known $\wp$-equation of Weierstrass [4]. Another point that should be mentioned here is the fact that algebraic integrability of equations (42) leads not merely to the $\theta$-function itself but to the elliptic $\mathfrak{L}_{x}$-divisor and even its non-canonical extension by the quadratic exponent. (Notice that constant $\kappa$ depends on modulus but constant $\boldsymbol{d}$ is free.) Further, the two-fold integration of the transcendental inversion operation in (51) can be reduced to integration of an algebraic function-our base operation.

COROLlary 8.3. The $\theta$-function can be defined through a meromorphic elliptic integral.

To prove this it will suffice to make the following substitution in formula (51):

$$
\int^{x} \Xi(y ; \boldsymbol{a}, \boldsymbol{b}, \boldsymbol{c}) d y=\int^{\Xi(x ; \boldsymbol{a}, \boldsymbol{b}, \boldsymbol{c})} \frac{z d z}{\sqrt{z(z-\boldsymbol{a})(z-\boldsymbol{b})}} .
$$


By this we obtain somewhat nonstandard way of introduction of the $\theta$-functions. To all appearances, Tikhomandritskiu [53] was the first to point out a way of definition of the $\theta$ through a meromorphic integral ${ }^{11}$ but his note [53] went unnoticed in the literature. He poses a question about the natural going from elliptic integrals to the theta-functions and presents the mode of transition between these transcendents by introducing the integral of the 2 nd kind elliptic integral. Indeed, rewriting formula (51) in the following form

$$
\theta(x)=\exp \int^{x}\left\{\int^{\Xi(x)} \frac{z d z}{\sqrt{z(z-\boldsymbol{a})(z-\boldsymbol{b})}}\right\} d x \cdot \mathrm{e}^{\kappa x^{2}+\boldsymbol{d} x+\boldsymbol{e}},
$$

we observe that such a way of introduction of a $\theta$-function is in effect the result of Liouvillian extension of a meromorphic integral, i. e., adjoining an exponent of integral of such an integral. By this means we may adopt this point as a differential definition of the $\theta$ à la Liouville and, subsequently, construct all the other objects of the theory: meromorphic (algebraic) functions are the $\theta$-ratios, Abelian integrals of 2nd kind are expressed through just introduced meromorphic integral (or, which is the same, the $\theta^{\prime}$ ), and the 3rd kind integrals are the logarithmic ratios of the $\theta$ 's with free parameters (the $\Lambda$-objects). Holomorphic integrals are of course the independent objects; they are not defined/determined through any other ones. An important role of a meromorphic integral was already observed by Clebsch \& Gordan in preface to their book [18, p. VI] on the base of Jacobi's formula

$$
\frac{\Theta(u)}{\Theta(0)}=\exp \int_{0}^{u} Z(u) d u
$$

where $Z(u)$ is a Jacobi zeta-function notation in the theory of elliptic functions [4].

The above differential properties of the $\Theta$ 's splittable to $\theta$ 's raise the question as to whether the general multi-dimensional $\Theta$-functions admit the similar 'differential kind' definition. In particular, whether exists the purely Liouvillian definition of an $x$-section $\Theta(x \boldsymbol{U}+D)$ like formula $(52)^{12}$ or, if any, the closed set of partial $D E s$ defining the complete set of the general $\Theta, \Theta^{\prime}(\boldsymbol{z})$-functions as ones of the $g$ arguments $\boldsymbol{z}$ ? Some relations between $\Theta$ 's and meromorphic Abelian integrals can be found in lectures by Weierstrass (though no really this point has been mentioned in the modern literature) but the question about closed and differentially Liouvillian definition (if it exists) of a $4^{g}$-set of the $g$-dimensional $\Theta$-functions and associated derivatives $\Theta^{\prime}$ remains an important open problem.

\footnotetext{
${ }^{11}$ He does not mention the quadratic extension and differential closedness of the set $\left\{\theta_{k}, \theta_{1}^{\prime}\right\}$, however.

${ }^{12}$ Roughly speaking, one needs an extensive strengthening of Theorem 6.1; whence it follows that

$$
-2 \ln _{x x} \Theta(x \boldsymbol{U}+\boldsymbol{D})=\ln _{x x} \Psi+\left(\ln _{x} \Psi\right)^{2}+\text { const }
$$

and, since the $\Psi$ is an exponent of the 3rd kind Abelian integral (formula (25)), that is $\Psi=\exp \mathbf{I I I}(\gamma$ 's $)$, the object $\Theta(x \boldsymbol{U}+\boldsymbol{D})$ itself is computed as a 'Liouvillian extension of Abelian integrals':

$$
\Theta(x \boldsymbol{U}+\boldsymbol{D})=\exp -\frac{1}{2}\left\{\boldsymbol{I I I}\left(\gamma^{\prime} \mathrm{s}\right)+\iint\left[\mathbf{I I I}\left(\gamma^{\prime} \mathrm{s}\right)\right]_{x}^{2} d x d x\right\} \mathrm{e}^{a x^{2}+b x+c}
$$
}

which is a reminiscence of formula (51). 


\section{Non-finite-gap integrable counterexamples}

Definition of integrability domain is not a subject of the $\Theta$-function techniques. Therefore we may generate integrable equations by any way differed from the classical FG-structure defined by formula (6) and Theorem 5.1. For example, equations coming no from operators $\widehat{\boldsymbol{L}}\left(\{U\} ; \partial_{x}\right)$ by taking the canonical spectral equation $\widehat{\boldsymbol{L}}\left(\{U\} ; \partial_{x}\right) \Psi=\lambda \Psi$ form in general the operator $\lambda$-pencils $\widehat{\boldsymbol{L}}\left(\{U\} ; \partial_{x}, \lambda\right) \Psi=0$, say, (20). Their solution structure is not known a priori ${ }^{13}$. Moreover, we can even construct an equation fitting no in the FG-scheme but having the same formal $\Theta$-function form of solution.

Example 6. Omitting in notation the elliptic modulus $\tau$, elucidate the said above by the following modification of the 2-gap Lamé equation:

$$
\Psi_{x x}=\{24 \wp(2 x)+8 \wp(2 x-\mathfrak{u})+16 \wp(\mathfrak{u})\} \Psi .
$$

It has a solution of the formal 2-gap Baker-Akhiezer form (44):

$$
\Psi(x ; \mathfrak{u})=\frac{d}{d x} \frac{\Lambda(x ; \mathfrak{u})}{\theta_{1}(x)}, \quad h=4 \zeta(\mathfrak{u})-2 \zeta(2 \mathfrak{u})
$$

(exercise: check this solution). By Theorems 7.1 and 8.2 this example is algebraically integrable over $\mathbb{C}_{\partial}(\wp(2 x), \wp(2 x-\mathfrak{u}))$ (and over $\mathbb{C}_{\partial}\left(\theta_{1}, \Lambda\right)$, of course) with solvable Galois group but it has little in common with commutative BurchnallChaundy operators, BA-function, or spectral lacunae. Formula (53) shows that this $\Psi$-function has no even a pole at point $2 x=\mathfrak{u}$ where potential does. This pole depends on a spectral parameter $\mathfrak{u}$ lying on an elliptic curve.

Nevertheless this example should not be considered as 'too artificial' because there exist the $\Theta$-function integrable models having algebraic curves with $x$-dependent branch-points. Ernst's equations in general relativity [36] provide a nice example along these lines.

9.1. Hermite's operator pencil. Consider now, in the framework of PicardVessiot theory, Hermitian equation (11) itself. It is not a Burchnall-Chaundy operator but the operator $\lambda$-pencil. By virtue of formulae (11)-(13), we define this pencil over field $\mathcal{N}([u])$ and repeat arguments about $\lambda$-dependence of $R$.

One of solutions to this pencil is not exponential but a purely Abelian meromorphic function (22), i. e., differential polynomial

$$
R_{1}=R([u] ; \lambda)=: \mathbf{P} \ni \mathcal{N}([u]) .
$$

Since base of solutions to Eq. (11) is $\left\{\Psi_{+}^{2}, \Psi_{+} \Psi_{-}, \Psi_{-}^{2}\right\}$ and $\Psi_{+}^{2} \notin \mathcal{N}([u])$, we may put the second solution as a square of the BA-function $R_{2}=\Psi_{+}^{2}$, where $\Psi_{+}$is an adjoint transcendent

$$
\Psi_{+}:=\exp \int^{x} \frac{\mathbf{P}_{x}+2 \mu}{2 \mathbf{P}} d x
$$

The third solution is $R_{3}=\Psi_{-}^{2}$ and we obtain that

$$
R_{1}=\mathbf{P}, \quad R_{2}=\Psi_{+}^{2}, \quad R_{3}=\frac{\mathbf{P}^{2}}{\Psi_{+}^{2}} .
$$

\footnotetext{
${ }^{13}$ It is well known, however, that Eq. (20) is related to a matrix canonical eigenvalue problem.
} 
Hence rationality domain is the same as in Theorem 3.4, that is $\mathcal{N}([u])\left\langle\Psi_{+}\right\rangle$, and this extension is a Liouvillian one of the transcendence degree 1 (we consider only the generic case $\lambda \neq E_{j}$ ). We therefore can obtain the following result.

THEOREM 9.1. Under the generic $\lambda \neq E_{j}$ the Galois group of Hermite's equation (11) defined over $\mathcal{N}([u])$ is connected and isomorphic to the diagonal group $\mathfrak{G}=\operatorname{Diag}\left(1, \alpha, \alpha^{-1}\right)$. Equations (11) is factorizable over field $\mathcal{N}([u])$.

PROOF. As in proof of Theorem 3.4 we perform a linear transformation of the basis $\left\{R_{1}, R_{2}, R_{3}\right\}$ and check invariance of the base differential relations between $R$ 's. Let us take relations of the zero and 1st order in derivatives:

$$
R_{1}=\mathbf{P}, \quad R_{2} R_{3}=\mathbf{P}^{2}, \quad\left(R_{2}\right)_{x}=\frac{\mathbf{P}_{x}+2 \mu}{\mathbf{P}} R_{2}, \quad\left(R_{3}\right)_{x}=\frac{\mathbf{P}_{x}-2 \mu}{\mathbf{P}} R_{3},
$$

which result from properties (54). Using condition $\mu \neq 0$, one easily derives that admissible transformations are

$$
R_{1} \mapsto 1 \cdot R_{1}, \quad R_{2} \mapsto \alpha \cdot R_{2}, \quad R_{3} \mapsto \delta \cdot R_{3}
$$

and $R_{2} R_{3} \mapsto 1 \cdot R_{2} R_{3}$. Hence $\delta=\alpha^{-1}$. Solutions $\left\{R_{1}, R_{2}, R_{3}\right\}$ are in general not algebraic functions, hence $\alpha$ is a free nonzero complex number and we do not need further to analyze the remaining relations of second order in derivatives of $R$ 's (they will be automatically satisfied). This yields a connectivity of the group and the matrix ${ }^{14} \operatorname{Diag}\left(1, \alpha, \alpha^{-1}\right)$. Factorizations of Eq. (11) are deducible by use of Liouville's scheme since we know solutions to equation. For example

$$
\begin{aligned}
\partial_{x x x}-4(u+\lambda) \partial_{x}-2 u_{x} & =\left(\partial_{x}+\frac{\mathbf{P}_{x}+2 \mu}{\mathbf{P}}\right) \partial_{x}\left(\partial_{x}-\frac{\mathbf{P}_{x}+2 \mu}{\mathbf{P}}\right) \\
& =\left(\partial_{x}+\frac{\mathbf{P}_{x}+2 \mu}{\mathbf{P}}\right)\left(\partial_{x}-\frac{2 \mu}{\mathbf{P}}\right)\left(\partial_{x}-\frac{\mathbf{P}_{x}}{\mathbf{P}}\right)
\end{aligned}
$$

These factorizations are not unique because equation has order 3.

9.2. Inversion of non-holomorphic integrals. As a last counterexample we consider an equation that leads, on the one hand, to a nonstandard case of the inversion problem, and, on the other hand, to a nonlinear $x$-evolution in a theta-function argument. As we shall see, there is no essential difference between (quadrature) integrability schemes of this example and those of pure FG-potentials. This example was already pointed out as non-standard in [54].

Example 7. Let us consider the following spectral problem

$$
\Psi_{x x}=\frac{\lambda}{v^{2}} \Psi
$$

where $v=v(x)$. As long as we have deal with invariant integration of (55) (Sect. 3), the theory has just non-essential modifications and we restrict ourselves to writing down all of its attributes in a form of references source for the simplest but nontrivial case $g=1$. We put

$$
R(x ; \lambda)=v \lambda-\phi(x), \quad \phi(x):=2 a(x-b)(x-c),
$$

\footnotetext{
${ }^{14}$ All this can also be seen from the fact that Galois group belongs to $\mathrm{SL}_{3}(\mathbb{C})$ and transcendence degree of the extension is unity.
} 
where $a, b, c \in \mathbb{C}$. Novikov's equation is the equation $v^{3} v_{x x x}-4 \phi v_{x}+4 \phi_{x} v=0$ and its integrals are as follows:

$$
\begin{gathered}
\mu^{2}=\lambda^{3}+3 E_{2}([v]) \lambda^{2}+E_{1}([v]) \lambda+a^{2}(b-c)^{2} \\
3 E_{2}=-\frac{1}{2} v v_{x x}+\frac{1}{4} v_{x}^{2}-2 \frac{\phi}{v}, \quad E_{1}=\frac{1}{2} \phi v_{x x}-\frac{1}{2} \phi_{x} v_{x}+\frac{\phi^{2}}{v^{2}}+2 a v .
\end{gathered}
$$

'Trace formula' follows from a direct analogy of (22), i. e., we set $R=(\lambda-\gamma) v$, but inversion problem becomes an inversion of the logarithmic integral rather than Jacobi problem (26). Indeed, manipulations with integrals $E_{1}, E_{2}$ show that

$$
\int^{\gamma} \frac{1}{z-E_{2}} \frac{d z}{\sqrt{4 z^{3}-g_{2} z-g_{3}}}=\frac{1}{2 a(b-c)} \ln \frac{x-b}{x-c}+D,
$$

where $g_{2}=12 E_{2}^{2}-4 E_{1}$ and $g_{3}=4 E_{1} E_{2}-8 E_{2}^{3}-a^{2}(b-c)^{2}$.

From (58) it follows that the $\theta$-function description undergoes changes since $x$-evolution on jacobian is essentially non-linear. We pass from parameter $E_{2}$ to $\varrho$ by the rule $\wp(2 \varrho)=E_{2}$ and represent the logarithmic integral (58) in terms of $\theta$-functions of the holomorphic one $\boldsymbol{r}: \gamma=\wp(2 \boldsymbol{r})$. We arrive at a transcendental equation determining function $\boldsymbol{r}=\boldsymbol{r}(x)$ :

$$
\ln \frac{\theta_{1}(\boldsymbol{r}-\varrho)}{\theta_{1}(\boldsymbol{r}+\varrho)}+2 \frac{\theta_{1}^{\prime}(\varrho)}{\theta_{1}(\varrho)} \boldsymbol{r}=\frac{\wp^{\prime}(2 \varrho)}{a(b-c)} \ln \frac{x-b}{x-c}+D .
$$

As a result we obtain that ultimate solution to the $\Psi$-function is far from obvious:

$$
\begin{gathered}
\Psi_{x x}=\frac{\lambda \wp^{2}(2 \boldsymbol{r})}{a^{2}(x-b)^{2}(x-c)^{2}} \Psi, \quad \Psi^{ \pm}(x ; \lambda)=\frac{\sqrt{(x-b)(x-c)}}{\sqrt{\wp(2 \boldsymbol{r})}} \cdot \frac{\Lambda(\boldsymbol{r} ; \pm \mathfrak{u})}{\theta_{1}(\boldsymbol{r})}, \\
\lambda=\wp(2 \mathfrak{u})-\wp(2 \varrho), \quad h=2 \eta \mathfrak{u}^{2} .
\end{gathered}
$$

A direct check of this solution is a good exercise in theta-calculus. Moreover, nonlinearity of this $x$-evolution is two-fold. Logarithm (58) contains a fractionlinear function but the principal nonlinearity comes from a transcendental nonlinearity of $\boldsymbol{r}(x)$. It never becomes linear even though we replace the general case in (56), that is $\phi(x)=2 a(x-b)(x-c)$, with the particular one $\phi(x)=$ const.

\section{Concluding remarks}

The properties of $\theta$-functions outlined above differ in a crucial respect from classical special functions since the latter ones are defined by ODEs not integrable in quadratures over elementary or algebraic functions. For example Bessel's functions or the Painlevé transcendents. Therefore when generalizing rational theory (solitons), not only do algebraic integrability takes place for Novikov's equations but it also takes place for linear spectral equations and even $\theta$-functions. In all these cases the integration procedure has been closed at a single and common step: adjunction of the inversion operation $\Xi$. The elementary theory does not get by without inversion as well:

$$
\int \text { rational functions } \rightarrow \ln \rightarrow \text { inversion } \rightarrow \text { exponent } \rightarrow \text { solitons. }
$$

It should be also emphasized that it makes no difference whether 1st kind integrals (Jacobi problem) or 2nd, 3rd kind ones have been inverted (see, e. g., (58)). The only thing is needed for the (Liouvillian) algebraic integrability: inversion of indefinite integrals of any algebraic functions. Roughly speaking, the inversion 
procedure appearing in 'theta-methods' has also the Liouvillian characterization because, according to important Eq. (16), adjunction of any kind Abelian integrals above is allowed. Nontrivial examples on inversion of meromorphic integrals can be found in monograph [28]; they are associated with the Camassa-Holm hierarchy and have also the theta-function description. In the same place quite extensive bibliography is presented. In other words, in regard to invariant integrability, the choice of the $\Theta$-series or the inversion operation $\Xi$ is just a question of nomenclature. Introducing $\Theta$ is equivalent to removing $\gamma$ 's from formulae like (23)-(25) and conversely. As for analytic representation of solutions, the $\Theta$-series is of course the fundamental object.

\section{References}

[1] Acosta-Humánez, P. B. Galoisian Approach to Supersymmetric Quantum Mechanics. The integrability analysis of the Schrodinger equation by means of differential Galois theory. Verlag Dr. Muller, 2010.

[2] Acosta-Humánez, P. B., Morales-Ruiz, J.-J. \& Weil, J.-A. Galoisian Approach to Integrability of the Schrödinger Equation. Rep. Math. Phys. 67(3) (2011), 305-374.

[3] Akniezer, N. I. A continuous analogue to orthogonal polynomials on a system of intervals. Soviet Math. Dokl. 2 (1961), 1409-1412.

[4] Akniezer, N. I. Elements of the Theory of Elliptic Functions. Amer. Math. Soc., Providence, RI, 1990.

[5] Al'Ber, S. I. Исследование уравнений Кортевега-де Фриза методом рекуррентных соотношений. (Investigation of equations of Korteweg-de Vries type by method of recurrence relations). Journ. London Math. Soc. (2) XIX(3) (1979), 467-480 (in Russian).

[6] Baker, H. F. Abelian Functions. Abel's theorem and the allied theory of theta functions. Cambridge Univ. Press, Cambridge, 1897.

[7] BAKer, H. F. Note on the foregoing paper, "Commutative ordinary differential operators," by J. L. Burchnall and J. W. Chaundy. Proc. Royal Soc. London A 118 (1928), 584-593.

[8] Belokolos, E. D., Bobenko, A. I., Enol'skit, V. Z., Its, A. R. \& Matveev, V. B. Algebro-Geometric Approach to Nonlinear Integrable Equations. Springer Series in Nonlinear Dynamics, Springer-Verlag, Berlin-Heidelberg-New York, 1994.

[9] Беркович, Л. М. Факторизация и преобразования дифференциалъных уравнений. РХД, Москва, 2002; Berкovich, L. М. Factorization and transformations of differential equations. RCD, Moscow, 2002 (in Russian).

[10] Braverman, A., Etingof, P. \& Gaitsgory, D. Quantum integrable systems and differential Galois theory. Transformation Groups 2(1) (1997), 31-56.

[11] Brezhnev, Yu. V. Dubrovin equations for finite-gap potentials. Russ. Math. Surveys $\mathbf{5 7}(2)$ (2002), 415-417.

[12] Брежнев, Ю. В. Исторические замечания $₹$ теории конечнозонного интегрирования. http://arXiv.org./nlin.SI/0504051 (in Russian); BreZhnev, Yu. V. Historical remarks to the theory of finite-gap integration.

[13] Brezhnev, Yu. V. What does integrability of finite-gap or soliton potentials mean? Phil. Trans. Royal Soc. Ser. A 366(1867) (2008), 923-945.

[14] Brezhnev, Yu. V. A $\tau$-function solution of the sixth Painlevé transcendent. Theor. Math. Phys. 160(3) (2009), 1615-1632.

[15] Buchstaber, V. M., Enolskit, V. Z. \& Leykin, D. V. Kleinian functions, hyperelliptic Jacobians and applications. Review in Mathematics and Mathematical Physics 10(2). Eds. S. Novikov \& I. Krichever. Gordon and Breach, London, (1997), pp. 1-125.

[16] Burchnall, J. L. \& Chaundy, T. W. Commutative Ordinary Differential Operators. Proc. Royal Soc. London. Ser. A 118(780) (1928), 557-583.

[17] Chudnovsky, D. V. \& Chudnovsky, G. V. Appendix I: Travaux de J. Drach (1919). Classical and Quantum Models and Ariphmetic Problems. Lecture Notes in Pure and Applied Mathematics. Eds. D. V. Chudnovsky \& G. V. Chudnovsky. Marcel Dekker, New York, 1984, pp. $445-453$. 
[18] Clebsch, A. \& Gordan, P. Theorie der Abelschen Functionen. Druck und Verlag von B. G. Teubner, Leipzig, 1866.

[19] Dickey, L. A. Solitons Equations and Hamiltonian Systems. 2nd edn. Advance Series in Math. Physics 26. World Scientific, Singapore, 2003.

[20] Drach, J. Détermination des cas de réduction de l'équation différentielle $\frac{d^{2} y}{d x^{2}}=[\varphi(x)+h] y$. Compt. Rend. Acad. Sci. 168 (1919), 47-50.

[21] Drach, J. Sur l'integration par quadratures de l'equation $\frac{d^{2} y}{d x^{2}}=[\varphi(x)+h] y$. Compt. Rend. Acad. Sci. 168 (1919), 337-340.

[22] Dubrovin, B. A. Inverse problem for periodic finite-zoned potentials in the theory of scattering. Funct. Anal. Appl. 9 (1975), 61-62.

[23] Dubrovin, B. A. Periodic problems for the Korteweg-de Vries equation in the class of finite band potentials. Funct. Anal. Appl. 9 (1975), 215-223.

[24] Dubrovin, B. A. Theta functions and non-linear equations. Russ. Math. Surveys 36(2) (1981), 11-92.

[25] Dubrovin, B. A., Matveev, V. B. \& Novikov, S. P. Non-linear equations of the Kortewegde Vries type, finite-zone linear operators and Abelian varieties. Russ. Math. Surveys 31(1) (1976), 59-146.

[26] Ehlers, F. \& KNÖRRER, H. An algebro-geometric interpretation of the Bäcklund transformation for the Korteweg-de Vries equation. Comment. Math. Helv. 57(1) (1982), 1-10.

[27] Gel'fand, I. M. \& Dikit, L. A. Asymptotic behaviour of the resolvent of Sturm-Liouville equations and the algebra of the Korteweg-de Vries equations. Russ. Math. Surveys 30(5) (1975), 77-113.

[28] Gesztesy, F. \& Holden, H. Soliton Equations and Their Algebro-Geometric Solutions. $(1+1)$-Dimensional Continuous Models. Cambridge studies in advanced mathematics $\mathbf{7 9}$, Cambridge University Press, Cambridge, 2003.

[29] Its, A. R. \& Matveev, V. B. Hill's operator with finitely many gaps. Funct. Anal. Appl. 9 (1975), 65-66.

[30] Its, A. R. \& Matveev, V. B. Schrödinger operators with finite-gap spectrum and N-soliton solutions of the Korteweg-de Vries equation. Theor. Math. Phys. 23(1) (1975), 343-355.

[31] Kaplansky, I. An introduction to differential algebra. Hermann, Paris, 1957.

[32] Kolchin, E. R. Algebraic matrix groups and the Picard-Vessiot theory of homogeneous linear ordinary differential equations. Annals of Math. 49 (1948), 1-42.

[33] Kolchin, E. R. Abelian Extensions of Differential Fields. Amer. J. Math. 82(4) (1960), 779790.

[34] Kolchin, E. R. Differential Algebra and Algebraic Groups. Academic Press, New York, 1973.

[35] Kovacic, J. J. An algorithm for solving second order linear homogeneous differential equations. Journ. Symbolic Comput. 2(1) (1986), 3-43.

[36] Korotkin, D. A. \& Matveev, V. B. Theta Function Solutions of the Schlesinger System and the Ernst Equation. Funct. Anal. Appl. 34(4) (2000), 252-264.

[37] Krichever, I. M. Methods of algebraic geometry in the theory of non-linear equations. Russ. Math. Surveys 32(6) (1977), 185-213.

[38] Krichever, I. M. Commutative rings of ordinary linear differential operators. Funct. Anal. Appl. 12 (1978), 175-185.

[39] LAX, P. D. Periodic Solutions of the KDV Equation. Comm. Pure and Appl. Math. XXVIII (1975), 141-188.

[40] Maier, R. S. Lamé polynomials, hyperelliptic reductions and Lamé band structure. Phil. Trans. Royal Soc. Ser. A 366(1867) (2008), 1115-1153.

[41] Matveev, V. B. Abelian functions and solitons. Preprint n. 373, Wrocław University, 1976, pp. 1-98.

[42] Matveev, V. B. Positons: slowly decreasing analogues of solitons. Theor. Math. Phys. 131(1) (2002), 483-497.

[43] Matveev, V. B. 30 years of finite-gap integration theory. Phil. Trans. Royal Soc. Ser. A 366(1867) (2008), 837-875.

[44] McKean, H. P. \& VAn Moerbeke, P. The spectrum of Hill's equation. Inventiones Mathematicae 30(3) (1975), 217-274.

[45] Morales-Ruiz, J.-J. Differential Galois theory and non-integrability of Hamiltonian systems. Progress in Mathematics 179, Birkhäuser Verlag, Basel, 1999. 
[46] Newell, A. C. Solitons in Mathematics and Physics. CBMS-NSF Regional Conference Series in Applied Mathematics 48, SIAM, Philadelphia, PA, 1985.

[47] Nijhoff, F., Hone, A. \& Joshi, N. On a Schwarzian PDE associated with the KdV hierarchy. Phys. Lett. A 267 (2000), 147-156.

[48] Novikov, S. P. The periodic problem for the Korteweg-de Vries equation I. Funct. Anal. Appl. 8(3) (1974), 236-246.

[49] Previato, E. Multivariable Burchnall-Chaundy theory. Phil. Trans. Royal Soc. Ser. A 366(1867) (2008), 1155-1177.

[50] van der Put, M. \& Singer, M. F. Galois Theory of Linear Differential Equations. SpringerVerlag, Berlin, 2003.

[51] Singer, M. F. Liouvillian solutions of nth order homogeneous linear differential equations. Amer. Journ. Math. 103(4) (1981), 661-682.

[52] Singer, M. F. Liouvillian solutions of linear differential equations with Liouvillian coeffcients. Journ. Symbolic Comput. 11(3) (1991), 251-273.

[53] Тихомандрицкій, М. А. Замптка о введеніи $\Theta$-функцій вг теорію эллиптическихљ функцій. Сообщенія и протоколы заседаній математическаго общества при ИмперАторскомъ Харьковскомъ Университеть (1883), Cер. I, 47-67. Tikhomandritskil̆, М. А. A note on introduction of $\Theta$-functions to the theory of elliptic functions. Communications and proceedings of Math. Soc. of the Khar'kov Imperial Univ. (1883), Ser. I, 47-67 (in Russian). Brief version of this note: TichomandRITZKy, M. Ueber das Umkehrproblem der elliptischen Integrale. Math. Ann. XXII(3) (1883), 450-454.

[54] Ustinov, N. V. \& Brezhnev, Yu. V. On the $\Psi$-function for finite-gap potentials. Russ. Math. Surveys 57(1) (2002), 165-167.

[55] Whittaker, E. T. \& Watson, G. N. A Course of Modern Analysis: An Introduction to the General Theory of Infinite Processes and of Analytic Functions, with an Account of the Principal Transcendental Functions. Cambridge University Press, Cambridge, 1996.

E-mail address: brezhnev@mail.ru 